TRANSACTIONS OF THE

AMERICAN MATHEMATICAL SOCIETY

Volume 355, Number 10, Pages 3947-3989

S 0002-9947(03)03046-0

Article electronically published on June 24, 2003

\title{
QUANDLE COHOMOLOGY AND STATE-SUM INVARIANTS OF KNOTTED CURVES AND SURFACES
}

\author{
J. SCOTT CARTER, DANIEL JELSOVSKY, SEIICHI KAMADA, LAUREL LANGFORD, \\ AND MASAHICO SAITO
}

Dedicated to Professor Kunio Murasugi for his 70th birthday

\begin{abstract}
The 2-twist spun trefoil is an example of a sphere that is knotted in 4-dimensional space. A proof is given in this paper that this sphere is distinct from the same sphere with its orientation reversed. Our proof is based on a state-sum invariant for knotted surfaces developed via a cohomology theory of racks and quandles (also known as distributive groupoids).

A quandle is a set with a binary operation - the axioms of which model the Reidemeister moves in classical knot theory. Colorings of diagrams of knotted curves and surfaces by quandle elements, together with cocycles of quandles, are used to define state-sum invariants for knotted circles in 3-space and knotted surfaces in 4-space.

Cohomology groups of various quandles are computed herein and applied to the study of the state-sum invariants. Non-triviality of the invariants is proved for a variety of knots and links, and conversely, knot invariants are used to prove non-triviality of cohomology for a variety of quandles.
\end{abstract}

\section{Introduction}

A quandle is a set with a self-distributive $((a * b) * c=(a * c) *(b * c))$ binary operation the axioms of which are partially motivated by classical knot theory. We derive a cohomology theory for quandles diagrammatically from Reidemeister moves for classical knots and knotted surfaces. Our definition of quandle (co)homology is a modification of rack (co)homology defined in [13] and [14]. Quandle cocycles are used to define state-sum invariants for knots and links in dimension 3 and for knotted surfaces in dimension 4 . As the main application of the invariant, we show that the invariant detects non-invertible knotted surfaces.

The invariants defined are demonstrated to be non-trivial in a variety of examples. In many cases, the invariant is related to linking numbers (Sections 8 and 9). In the case of a 3-component surface link, there is a notion of 3-fold linking, defined combinatorially, that can be used to compute the invariant over trivial quandles (Section 9). In the classical case of knotted curves, it is shown that the trefoil $\left(3_{1}\right.$ in the tables) and the figure 8 knot $\left(4_{1}\right.$ in the tables) have non-trivial (mod 2$)$-cocycle invariants over a 4-element quandle associated to the rotations of a tetrahedron.

Received by the editors August 21, 2001 and, in revised form, February 20, 2002.

2000 Mathematics Subject Classification. Primary 57M25, 57Q45; Secondary 55N99, 18 G99.

Key words and phrases. Knots, links, knotted surfaces, quandle, rack, quandle cohomology, state-sum invariants, non-invertibility. 
Conversely, knots are used to prove algebraic results - non-triviality of cohomology groups for a variety of quandles. As a main topological application, the 2-twist spun trefoil is shown (Section 11) to be non-invertible, i.e., distinct from itself with the reversed orientation, by evaluating the state-sum invariant with a 3-cocycle over the 3 -element dihedral quandle (defined below). After a preliminary version of this paper was circulated, Rourke and Sanderson [39] provided a much shorter proof of non-invertibility. Furthermore, Satoh and Shima [46] have found other topological applications of the invariant.

In [13] and [14], the general framework for defining invariants from racks and quandles and their homology and cohomology is outlined. The present paper defines knot invariants by means of a state-sum, using quandle cocycles. This cocycle invariant can also be seen as an analogue of the Dijkgraaf-Witten invariants for 3manifolds [9] in that colorings and cocycles are used to define state-sum invariants. Another analogue of the Dijkgraaf-Witten invariants was applied to triangulated 4-manifolds in [5]. The non-invertibility for certain classical knots had long been presumed since the 1920s but proved first by Trotter in the 1960s and later using hyperbolic structures (see [21, 28, 31]). Fox [15] presented a non-invertible knotted sphere using Alexander modules. Alexander modules, however, fail to detect noninvertibility of the 2-twist spun trefoil. In this paper we show its non-invertibility using the cocycle state-sum invariants. In particular, the cocycle invariants are the first state-sum invariants in dimension 4 that carry information not contained in the Alexander modules. It was pointed out to us by D. Ruberman that Farber-Levine pairings [10, 11, 34] and Casson-Gordon invariants detect non-invertibility of some twist-spun knots [22, 42]. A. Kawauchi pointed out that our invariant detects the non-invertibility of the twist-spun trefoil even after adding trivial 1-handles, thus increasing the genus of the surface. Therefore, our invariant implies the new topological results, that these higher genus surfaces are non-invertible. He also informed us that Farber-Levine pairings are generalized for knotted surfaces of higher genus [30] (cf. [47]) by use of his duality [29], and that the (Farber-Levine-Kawauchi) pairings [30] also detect non-invertibility of higher genus surfaces. Thus, relations between these invariants and the state-sum invariants deserve investigation.

Our inspiration for the definition of these invariants is found in Neuchl's paper [37] where related cocycles are used to give examples of representations of a Hopf category in a braided monoidal 2-category using quantum groups of finite groups. Our definition was derived from an attempt to construct a 2-functor from the braided 2-category of knotted surfaces as summarized in [1] and presented in detail in 2, to another 2-category constructed from quandles.

1.1. Organization. Section 2 contains the basic definitions of racks and quandles. Rack cohomology and quandle cohomology are defined in Section 3. Section 4 defines invariants of classical knots and links via assigning 2-cocycles to crossings. Section [5 contains the analogous definition for knotted surfaces. Section 6 presents calculations of cohomology groups for some exemplary quandles. Section 7 relates the quandle 2-cocycles to group 2-cocycles when the quandle is a group with conjugation as the operation. Section 8 contains computations in the case of classical knots and links. Section 9 defines a notion of linking for knotted surfaces. This linking is used to exemplify non-triviality of the state-sum invariant in the case of surfaces in 4-space. Section 10 develops techniques for computation for surface 


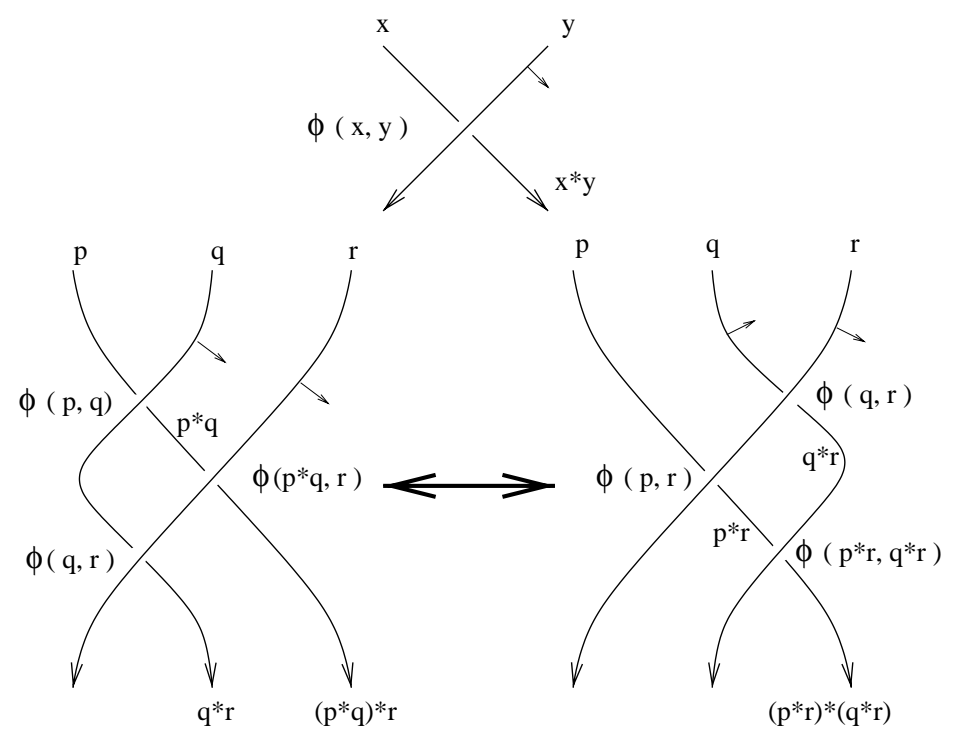

Figure 1. Type III move and the quandle identity

braids. In Section 11 these techniques are applied to the 2-twist spun trefoil and its orientation reversed image to demonstrate that these knotted surfaces are distinct.

\section{RACKS, QUANDLES, AND KNOTS}

A quandle, $X$, is a set with a binary operation $(a, b) \mapsto a * b$ such that:

(I) For any $a \in X, a * a=a$.

(II) For any $a, b \in X$, there is a unique $c \in X$ such that $a=c * b$.

(III) For any $a, b, c \in X$, we have $(a * b) * c=(a * c) *(b * c)$.

A rack is a set with a binary operation that satisfies (II) and (III).

A typical example of a quandle is a group $X=G$ with $n$-fold conjugation as the quandle operation: $a * b=b^{-n} a b^{n}$. Racks and quandles have been studied in, for example, [3, 12, 20, 27, 35.

The axioms for a quandle correspond respectively to the Reidemeister moves of type I, II, and III (see [12, [27, for example). Indeed, knot diagrams were one of the motivations to define such an algebraic structure. In all of our diagrams, orientations and co-orientations (normal vectors to the given diagram) are mutually determined by a right-hand rule. So tangent plus normal agrees with the counterclockwise orientation of the plane that contains the knot diagram. At a crossing of a classical knot diagram (in which the arcs are co-oriented), the under-arc is labeled on one segment by a quandle element, $x$, and along the other segment by the quandle product $x * y$ where $y$ is the quandle element labeling the overarc. The co-orientation can be used as a mnemonic for the multiplication; the arc towards which the normal of the over-arc points receives the product. See the top of Figure1. The choice of rack multiplication corresponds to the Wirtinger relation in the fundamental group: $x * y=y^{-1} x y$. The labels in the figure involving $\phi$, which are assigned to the crossings, will be used later.

Oriented knotted surface diagrams are co-oriented by the same rule (the orientation of the tangent plus the normal vector defines the given orientation of 3-space); 
conversely, co-orientations of knotted surface diagrams determine orientations. The co-orientation is denoted on the complement of the branch point set by means of a short normal arrow. In some of the illustrations only orientations are indicated, in some only co-orientations are indicated, and in some neither are indicated.

A function $f: X \rightarrow Y$ between quandles or racks is a homomorphism if $f(a * b)=$ $f(a) * f(b)$ for any $a, b \in X$.

\section{Cohomology of QUANDles AND KNOT DiAgRAmS}

We define the homology and cohomology theory for racks and quandles.

Let $C_{n}^{\mathrm{R}}(X)$ be the free abelian group generated by $n$-tuples $\left(x_{1}, \ldots, x_{n}\right)$ of elements of a quandle $X$. Define a homomorphism $\partial_{n}: C_{n}^{\mathrm{R}}(X) \rightarrow C_{n-1}^{\mathrm{R}}(X)$ by

$$
\begin{aligned}
\partial_{n}\left(x_{1}, x_{2}, \ldots, x_{n}\right) & \\
& =\sum_{i=2}^{n}(-1)^{i}\left[\left(x_{1}, x_{2}, \ldots, x_{i-1}, x_{i+1}, \ldots, x_{n}\right)\right. \\
& \left.-\left(x_{1} * x_{i}, x_{2} * x_{i}, \ldots, x_{i-1} * x_{i}, x_{i+1}, \ldots, x_{n}\right)\right]
\end{aligned}
$$

for $n \geq 2$ and $\partial_{n}=0$ for $n \leq 1$. Then $C_{*}^{\mathrm{R}}(X)=\left\{C_{n}^{\mathrm{R}}(X), \partial_{n}\right\}$ is a chain complex.

Let $C_{n}^{\mathbf{D}}(X)$ be the subset of $C_{n}^{\mathrm{R}}(X)$ generated by $n$-tuples $\left(x_{1}, \ldots, x_{n}\right)$ with $x_{i}=x_{i+1}$ for some $i \in\{1, \ldots, n-1\}$ if $n \geq 2$; otherwise, let $C_{n}^{\mathbf{D}}(X)=0$. If $X$ is a quandle, then $\partial_{n}\left(C_{n}^{\mathbf{D}}(X)\right) \subset C_{n-1}^{\mathbf{D}}(X)$ and $C_{*}^{\mathbf{D}}(X)=\left\{C_{n}^{\mathbf{D}}(X), \partial_{n}\right\}$ is a subcomplex of $C_{*}^{\mathbf{R}}(X)$. Put $C_{n}^{\mathrm{Q}}(X)=C_{n}^{\mathrm{R}}(X) / C_{n}^{\mathrm{D}}(X)$ and $C_{*}^{\mathrm{Q}}(X)=\left\{C_{n}^{\mathrm{Q}}(X), \partial_{n}^{\prime}\right\}$, where $\partial_{n}^{\prime}$ is the induced homomorphism. Henceforth, all boundary maps will be denoted by $\partial_{n}$.

For an abelian group $A$, define the chain and cochain complexes

$$
\begin{aligned}
C_{*}^{\mathrm{W}}(X ; A)=C_{*}^{\mathrm{W}}(X) \otimes A, & \partial=\partial \otimes \mathrm{id} ; \\
C_{\mathrm{W}}^{*}(X ; A)=\operatorname{Hom}\left(C_{*}^{\mathrm{W}}(X), A\right), & \delta=\operatorname{Hom}(\partial, \mathrm{id})
\end{aligned}
$$

in the usual way, where $\mathrm{W}=\mathrm{D}, \mathrm{R}, \mathrm{Q}$.

3.1. Definition. The $n$th rack homology group and the $n$th rack cohomology group 13 of a rack/quandle $X$ with coefficient group $A$ are

$$
H_{n}^{\mathrm{R}}(X ; A)=H_{n}\left(C_{*}^{\mathrm{R}}(X ; A)\right), \quad H_{\mathrm{R}}^{n}(X ; A)=H^{n}\left(C_{\mathrm{R}}^{*}(X ; A)\right) .
$$

The $n$th degeneration homology group and the $n$th degeneration cohomology group of a quandle $X$ with coefficient group $A$ are

$$
H_{n}^{\mathbf{D}}(X ; A)=H_{n}\left(C_{*}^{\mathbf{D}}(X ; A)\right), \quad H_{\mathrm{D}}^{n}(X ; A)=H^{n}\left(C_{\mathrm{D}}^{*}(X ; A)\right) .
$$

The $n$th quandle homology group and the $n$th quandle cohomology group of a quandle $X$ with coefficient group $A$ are

$$
H_{n}^{\mathrm{Q}}(X ; A)=H_{n}\left(C_{*}^{\mathrm{Q}}(X ; A)\right), \quad H_{\mathrm{Q}}^{n}(X ; A)=H^{n}\left(C_{\mathrm{Q}}^{*}(X ; A)\right) .
$$

The cycle and boundary groups (resp. cocycle and coboundary groups) are denoted by $Z_{n}^{\mathrm{W}}(X ; A)$ and $B_{n}^{\mathrm{W}}(X ; A)\left(\operatorname{resp} . Z_{\mathrm{W}}^{n}(X ; A)\right.$ and $\left.B_{\mathrm{W}}^{n}(X ; A)\right)$, so that

$$
H_{n}^{\mathrm{W}}(X ; A)=Z_{n}^{\mathrm{W}}(X ; A) / B_{n}^{\mathrm{W}}(X ; A), H_{\mathrm{W}}^{n}(X ; A)=Z_{\mathrm{W}}^{n}(X ; A) / B_{\mathrm{W}}^{n}(X ; A)
$$

where $\mathrm{W}$ is one of $\mathrm{D}, \mathrm{R}, \mathrm{Q}$. We will omit the coefficient group $A$ if $A=\mathbf{Z}$ as usual.

Here we are almost exclusively interested in quandle homology or cohomology. So we drop the superscript/subscript $\mathrm{W}=\mathrm{Q}$ from the notation, unless it is needed. 
3.2. Remark. Recall that $C_{n}^{\mathrm{D}}(X ; A)$ is the subgroup of $C_{n}^{\mathrm{R}}(X ; A)$ generated by $\vec{x}=\left(x_{1}, \ldots, x_{n}\right) \in C_{n}^{\mathrm{R}}(X ; A)$ such that $x_{j}=x_{j+1}$ for some $j=1, \ldots, n-1$. Let $P^{n}(X ; A)=\left\{f \in C_{\mathrm{R}}^{n}(X ; A) \mid f(\vec{x})=0\right.$ for all $\left.\vec{x} \in C_{n}^{\mathbf{D}}(X)\right\}$; this set can be identified with $C_{\mathrm{Q}}^{n}(X ; A)$. (The set $P^{3}$ is related to branch points of knotted surface diagrams.) Then the quandle cohomology group is described as

$$
H_{\mathrm{Q}}^{n}(X ; A)=\left(P^{n}(X ; A) \cap Z_{\mathrm{R}}^{n}(X ; A)\right) / \delta\left(P^{n-1}(X ; A)\right) .
$$

There is another cohomology group defined by

$$
H_{\mathrm{Q}}^{n}(X ; A)^{\prime}=\left(P^{n}(X ; A) \cap Z_{\mathrm{R}}^{n}(X ; A)\right) /\left(P^{n}(X ; A) \cap B_{\mathrm{R}}^{n}(X ; A)\right) .
$$

This cohomology group makes sense even for a rack $X$. Studies of this cohomology group, in relation to the cohomology group $H_{\mathrm{Q}}^{n}(X ; A)$ and branch points, are expected.

3.3. Examples. The cocycle conditions are related to moves on knots and higher dimensional knots as indicated in Figures 1 [2 and 3. A 2-cocycle $\phi$ satisfies the relation

$$
\phi(p, r)+\phi(p * r, q * r)=\phi(p, q)+\phi(p * q, r) .
$$

And a 3 -cocycle $\theta$ satisfies the relation

$$
\begin{aligned}
& \theta(p, q, r)+\theta(p * r, q * r, s)+\theta(p, r, s) \\
& \quad=\theta(p * q, r, s)+\theta(p, q, s)+\theta(p * s, q * s, r * s) .
\end{aligned}
$$

In subsequent sections, such cocycles will be assigned to crossings of classical diagrams or triple points of knotted surface diagrams, respectively. Figure 1 shows that the sum of cocycles evaluated on quandle elements around the crossings of a diagram remains invariant under a Reidemeister type III move. The corresponding move for knotted surfaces (right-bottom of Figure 7), called the tetrahedral move, with choices of a height function and crossing information, is depicted in Figures 2 and 3 Although such figures involve four straight lines as cross sections of four planes in space, in Figures 2 and 3 we depicted curved lines instead, to make the figures look nicer. A 3-cocycle is assigned to each type III move in the figures; these moves correspond to triple points of a knotted surface diagram. Thus the sum of 3 -cocycles (evaluated on the quandle elements near the triple point) remains invariant under this move. Hence the cocycles can be used to define knot invariants. We turn now to a rigorous definition of such invariants.

\section{Cocycle invariants of Classical KnOtS}

4.1. Definition. A coloring on an oriented classical knot diagram is a function $\mathcal{C}: R \rightarrow X$, where $X$ is a fixed quandle and $R$ is the set of over-arcs in the diagram, satisfying the condition depicted in the top of Figure 1. In the figure, a crossing with over-arc, $r$, has color $\mathcal{C}(r)=y \in X$. The under-arcs are called $r_{1}$ and $r_{2}$ from top to bottom; the normal of the over-arc $r$ points from $r_{1}$ to $r_{2}$. Then it is required that $\mathcal{C}\left(r_{1}\right)=x$ and $\mathcal{C}\left(r_{2}\right)=x * y$.

Note that locally the colors do not depend on the orientation of the under-arc. The quandle element $\mathcal{C}(r)$ assigned to an $\operatorname{arc} r$ by a coloring $\mathcal{C}$ is called a color of the arc. This definition of colorings on knot diagrams has been known; see [12, 15] for example.

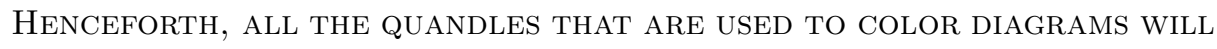
BE FINITE. 


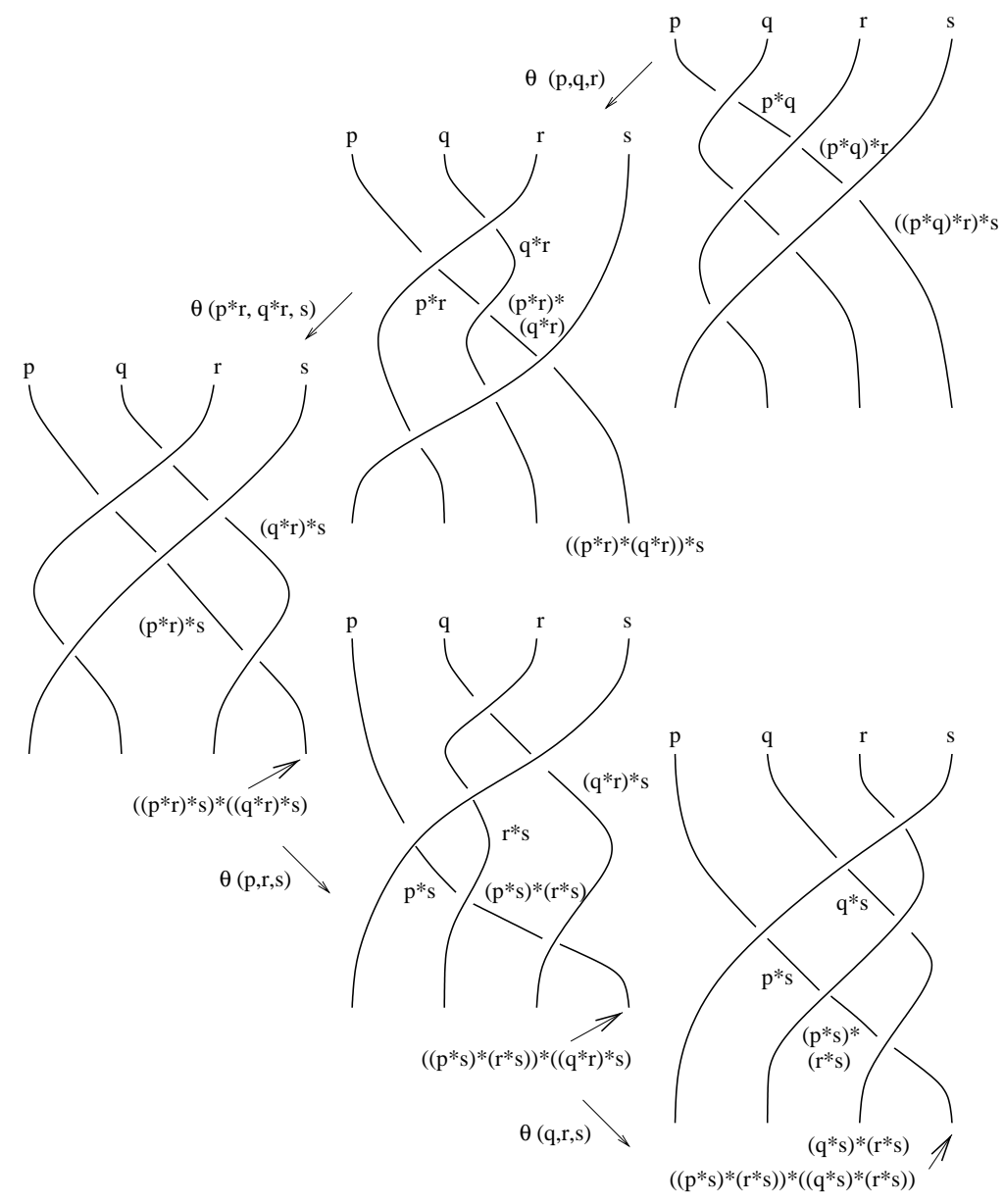

Figure 2. The tetrahedral move and a cocycle relation, LHS

At a crossing, if the pair of the co-orientation of the over-arc and that of the under-arc matches the (right-hand) orientation of the plane, then the crossing is called positive; otherwise it is negative. In Figure 4 the two possible oriented and co-oriented crossings are depicted. The left is a positive crossing, and the right is negative.

In what follows in this section, we suppose that a finite quandle $X$ which is used for colorings and an abelian coefficient group $A$ are fixed.

4.2. Definition. Let $\phi \in Z_{\mathrm{Q}}^{2}(X ; A)$ be a 2-cocycle. A (Boltzmann) weight, $B(\tau, \mathcal{C})$ (associated with $\phi$ ), at a crossing $\tau$ is defined as follows. Let $\mathcal{C}$ denote a coloring. Let $r$ be the over-arc at $\tau$, and $r_{1}, r_{2}$ be under-arcs such that the normal to $r$ points from $r_{1}$ to $r_{2}$. Let $x=\mathcal{C}\left(r_{1}\right)$ and $y=\mathcal{C}(r)$. Then define $B(\tau, \mathcal{C})=\phi(x, y)^{\epsilon(\tau)}$, where $\epsilon(\tau)=1$ or -1 , if the sign of $\tau$ is positive or negative, respectively. 



Figure 3. The tetrahedral move and a cocycle relation, RHS

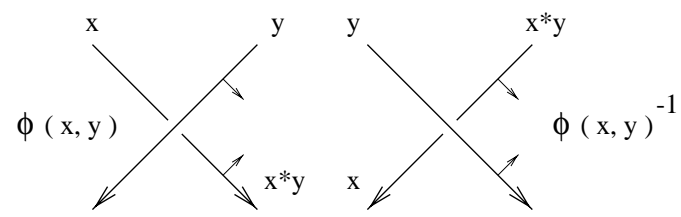

FiguRE 4. Weights for positive and negative crossings

4.3. Definition. Let $\phi \in Z_{\mathrm{Q}}^{2}(X ; A)$ be a 2-cocycle. The partition function, or a state-sum (associated with $\phi$ ), of a knot diagram is the expression

$$
\sum_{\mathcal{C}} \prod_{\tau} B(\tau, \mathcal{C})
$$

The product is taken over all crossings of the given diagram, and the sum is taken over all possible colorings. (The value of $B(\tau, \mathcal{C})$ is in the coefficient group $A$ written 


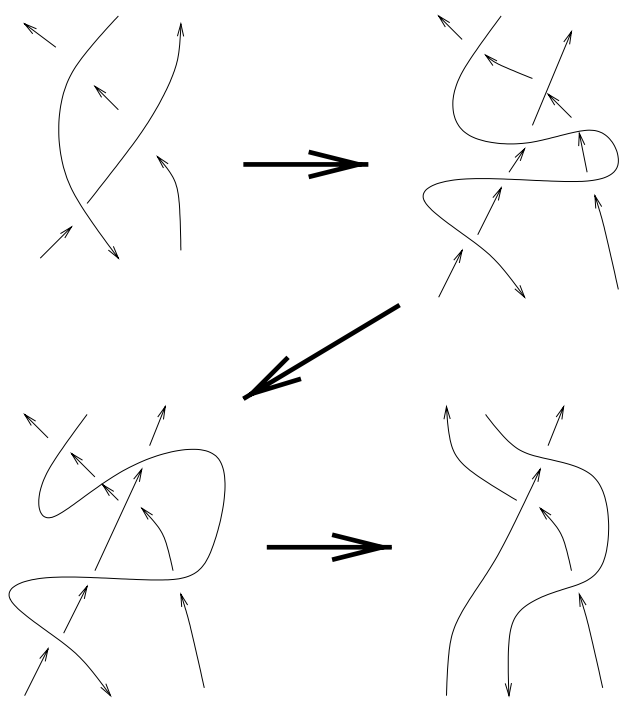

Figure 5. A type III move with different crossings

multiplicatively.) The formal sum is taken over all colorings, and hence the values of the state-sum are in the group ring $\mathbf{Z}[A]$.

4.4. Theorem. Let $\phi \in Z_{\mathrm{Q}}^{2}(X ; A)$ be a 2-cocycle. The partition function (associated with $\phi)$ of a knot diagram is invariant under Reidemeister moves, so that it defines an invariant of knots and links. Thus it will be denoted by $\Phi(K)$ (or $\Phi_{\phi}(K)$ to specify the 2-cocycle $\phi$ used).

Proof. There is a one-to-one correspondence between colorings before and after each Reidemeister move. Hence we check that the state-sum remains unchanged under Reidemeister moves for each coloring. For the type I move, the weight assigned to the crossing is of the form $\phi(x, x)^{ \pm 1}$, which is 1 by assumption that $\phi$ is a quandle cocycle. Thus the state-sum is invariant under type I moves. Recall from [27], there are two types of type II moves depending on whether the arcs are oriented in the same direction or different directions. In either case, at the two crossings of a type II move, the 2-cocycle weights are the same, but with opposing exponents. Therefore the weights cancel in the state-sum and the partition function is invariant under type II moves.

The definition of cocycles was formulated so that the partition function would be invariant under the type III move depicted in Figure 1, There are other possible type III moves depending on the signs of the crossings and the orientation of the edges of the central triangle. In [27, page 81, Kauffman presents the sketch of the argument which shows that the type III move with differently oriented triangle follows from the type II moves and one choice of type III move. In Figure 5 , we indicate how to change the sign of one of the crossings via an analogous technique. This shows invariance under all type III moves. See also [48] or [49].

4.5. Proposition. Let $\phi, \phi^{\prime} \in Z_{\mathrm{Q}}^{2}(X ; A)$ be 2-cocycles. If $\Phi_{\phi}$ and $\Phi_{\phi^{\prime}}$ denote the state-sum invariants defined from cohomologous cocycles $\phi$ and $\phi^{\prime}$ (so that $\phi=\phi^{\prime} \delta \psi$ for some 1-cochain $\psi)$, then $\Phi_{\phi}=\Phi_{\phi^{\prime}}$ (so that $\Phi_{\phi}(K)=\Phi_{\phi^{\prime}}(K)$ for any link $\left.K\right)$. 
In particular, the state-sum is equal to the number of colorings of a given knot diagram if the 2-cocycle used for the Boltzmann weight is a coboundary.

Proof. We prove the second half, as the first half follows from a similar argument. Suppose that $\phi(x, y)=\psi(x) \psi(x * y)^{-1}$, so that the cocycle is a coboundary. Pick a coloring of the diagram. We can think of the weight as a weight of the ends of each under-arc where the "bottom" end of a positive crossing receives the weight $\psi(x)$ while the top end of the under-arc receives a weight of $\psi(x * y)^{-1}$. The negativecrossing case is similar. The under-arc has only one color associated to it, so for a given arc, the weights at its two ends cancel. A given term in the state-sum then contributes a term of 1 to the state-sum. Thus when we sum over all colorings, we end up counting the colorings.

We say that the state-sum invariant of a knot/link $K$ is trivial if it is an integer. In this case, the integer is equal to the number of colorings of a diagram of $K$ by $X$.

\section{Cocycle invariants of KnOtTed SuRfaces}

First we recall the notion of knotted surface diagrams. See 7] for details and examples. Let $f: F \rightarrow \mathbf{R}^{4}$ denote a smooth embedding of a closed surface $F$ into 4-dimensional space. Such an embedding $f$, or its image $f(F)$, is called a knotted surface. By deforming the map $f$ slightly by an ambient isotopy of $\mathbf{R}^{4}$ if necessary, we may assume that $p \circ f$ is a general position map, where $p: \mathbf{R}^{4} \rightarrow \mathbf{R}^{3}$ denotes the orthogonal projection onto an affine subspace which does not intersect $f(F)$. Along the double curves, one of the sheets (called the over-sheet) lies farther than the other (under-sheet) with respect to the projection direction. The under-sheets are coherently broken in the projection, and such broken surfaces are called knotted surface diagrams.

When the surface is oriented, we take normal vectors $\vec{n}$ to the projection of the surface such that the triple $\left(\vec{v}_{1}, \vec{v}_{2}, \vec{n}\right)$ matches the orientaion of 3 -space, where $\left(\vec{v}_{1}, \vec{v}_{2}\right)$ defines the orientation of the surface. Such normal vectors are defined on the projection at all points other than the isolated branch points.

We fix a finite quandle $X$ and an abelian group $A$.

5.1. Definition. A coloring on an oriented (broken) knotted surface diagram is a function $\mathcal{C}: R \rightarrow X$, where $R$ is the set of regions in the broken surface diagram, satisfying the following condition at the double point set.

At a double point curve, two coordinate planes intersect locally. One is the over-sheet $r$, the other is the under-sheet, and the under-sheet is broken into two components, say $r_{1}$ and $r_{2}$. A normal of the over-sheet $r$ points to one of the components, say $r_{2}$. If $\mathcal{C}\left(r_{1}\right)=x \in X, \mathcal{C}(r)=y$, then we require that $\mathcal{C}\left(r_{2}\right)=x * y$. The quandle elements $\mathcal{C}(r)$ assigned to an arc $r$ by a coloring is called a color of $r$. See Figure [ 6 left.

\subsection{Lemma. The above condition is compatible at each triple point.}

Proof. The meaning of this lemma is as follows. There are 6 double curves near a triple point, giving 6 conditions on colors assigned. It can be checked in a straightforward manner that these conditions do not contradict each other. In particular, there is one of the 4 pieces of the lower sheet that receives color $(a * b) * c$ or 

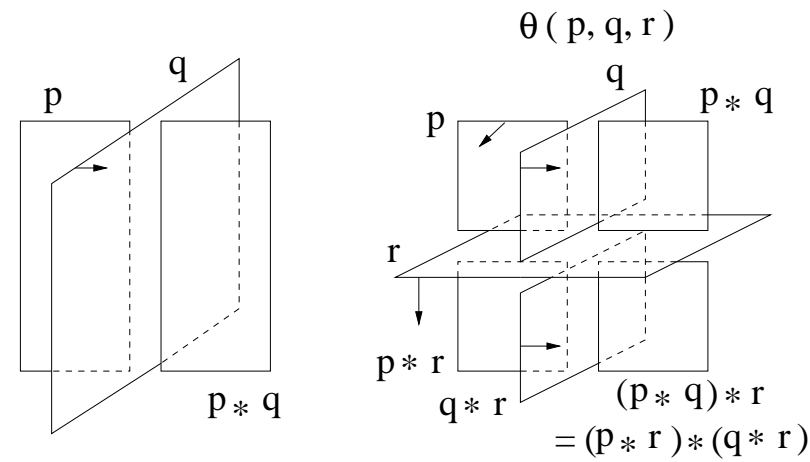

Figure 6. Colors at a double curve and a triple point

$(a * c) *(b * c)$ depending on what path was followed to compute the color. Since these values agree in the quandle, there is no contradiction. Figure 6 illustrates the situation.

5.3. Definition. Note that when three sheets form a triple point, they have relative positions top, middle, bottom with respect to the projection direction of $p: \mathbf{R}^{4} \rightarrow$ $\mathbf{R}^{3}$. The sign of a triple point is positive if the normals of top, middle, bottom sheets in this order match the orientation of the 3 -space. Otherwise, the sign is negative. We use the right-hand rule convention for the orientation of 3 -space. This definition is found, for example, in [7].

5.4. Definition. Fix a 3-cocycle $\theta \in Z_{\mathrm{Q}}^{3}(X ; A)$. A (Boltzmann) weight at a triple point, $\tau$, is defined as follows. Let $R$ be the octant from which all normal vectors of the three sheets point outwards; let a coloring $\mathcal{C}$ be given. Let $p, q, r$ be colors of the bottom, middle, and top sheets respectively, that bound the region $R$. Let $\epsilon(\tau)=1$ or -1 if $\tau$ is positive or negative, respectively. Then the Boltzman weight $B(\tau, \mathcal{C})$ at $\tau$ with respect to $\mathcal{C}$ is defined to be $\theta(p, q, r)^{\epsilon(\tau)}$ where $p, q, r$ are colors described above. Figure 6 illustrates the situation.

5.5. Definition. Let $\theta \in Z_{\mathrm{Q}}^{3}(X ; A)$ be a 3 -cocycle. The partition function, or a state-sum, (associated with $\theta$ ) of a knotted surface diagram is the expression

$$
\sum_{\mathcal{C}} \prod_{\tau} B(\tau, \mathcal{C})
$$

The product is taken over all triple points of the diagram, and the sum is taken over all possible colorings. As in the classical case, $A$ is written multiplicatively and the state-sum is an element of the group ring $\mathbf{Z}[A]$.

5.6. Theorem. We fix a 3 -cocycle $\theta \in Z_{\mathrm{Q}}^{3}(X ; A)$. The partition function does not depend on the choice of knotted surface diagram. Thus it is an invariant of knotted surfaces $F$, and denoted by $\Phi(F)$ (or $\Phi_{\theta}(F)$ to specify the 3-cocycle $\theta$ used).

Proof. Roseman provided analogues of the Reidemeister moves as moves to knotted surface diagrams and these analogous moves (called Roseman moves) are depicted in Figure [ [7, 40]. Note that in this figure projections are depicted, instead of broken surface diagrams. There are moves for all possible crossing information for the 



FigURE 7. Roseman moves for knotted surface diagrams

sheets involved in each move. Thus two knotted surface diagrams represent isotopic knotted surfaces if and only if the diagrams are related by a finite sequence of moves, called Roseman moves, taken from this list. There is a one-to-one correspondence between colorings before and after each Roseman move, so we check that the statesum is invariant under each Roseman move. However, the state-sum depends only on triple points, so we need only consider those moves that involve triple points. These moves are: (1) the creation or cancellation of a pair of oppositely signed triple points (indicated in the last column of the second row of the illustration); (2) moving a branch point through a sheet (third row, first column); and (3) the tetrahedral move (on the bottom right) that motivated the definition of the cocycles. 
In the first case, the pair of triple points have opposite signs, so for a given coloring, the two contributing factors of the state-sum cancel. In the second case, the branch point occurs on either the bottom/middle sheet or on the top/middle sheet, and these sheets have the same color. Since the weighting of the proximate triple point is a quandle cocycle ( $\operatorname{so} \theta(x, x, y)=\theta(x, y, y)=1)$, this factor does not contribute to the state-sum.

In the third case, there are several possible tetrahedral moves to consider that depend on (a) the local orientation of the sheets around the tetrahedron, and (b) the signs of the triple points that are the vertices of the tetrahedron. The definition of the cocycles and the illustrations Figure 2 and Figure 3 indicate that the state-sum is invariant under one of these possible choices. We will move a given tetrahedral move so that the planes involved coincide with planes in this standard position, but have possibly differing crossings or orientations. Then we generalize Turaev's technique to dimension 4 to show that the given move follows from the fixed move and invariance under adding or subtracting a cancelling pair of triple points.

Let $T_{0}$ be the four planes together with the choice of orientations depicted in Figures 2 and 3 and let $T$ be a given situation of four planes. Each sheet has relative height in 4-dimensions. Call them 1st, 2nd, 3rd, and 4th sheet from bottom to top, respectively, so that the 1st sheet is the bottom sheet and the 4th is top. In other words, the 4th sheet is unbroken, and the 1st sheet is broken into seven pieces in the broken surface diagram. Suppose that $T_{0}$ has $x y, x z, y z$ planes as 4 th, 3rd, and the 2 nd sheets respectively, and $x+y+z=1$ as the 1 st plane. We can isotope $T$ to $T_{0}$ in such a way that the 4 th sheets match with orientations. (In other words, isotope the 4th sheet of $T$ to that of $T_{0}$ so that the co-orientation normals also match.) By further isotopy match the 3rd sheets together with their normals. Then match the 2nd sheets. However, the normals may not match here (if the sign of the triple point among the sheets 2, 3, 4 is opposite). By isotopy, the 1st sheet of $T$ is one of the planes $\pm x \pm y \pm z=1$. However, there are two cases (before/after) of positions for a given tetrahedral move, so that we may assume that the 1st plane is one of four: $\pm x \pm y+z=1$. Thus we have four possibilities of orientation choices, those for 2nd and the 1st sheets, and four possibilities for the position of the bottom (1st) sheet.

Consider the case where the 1st sheet has the opposite orientation of the fixed situation in $T_{0}$. Then comparing to $T_{0}$, the signs of the triple points involving the 1st sheet reverse, and those for the triple point not having the sheet 1 remain the same. The former are the triple points among the sheets (123), (134), (124), and the latter is (234). However, recall that the cocycle assigned to the triple point (234) is $\theta(q, r, s)$ and appears in both sides of the move, and the rest of the terms are inverses of the 2-cocycle condition. Therefore the state-sum remains invariant in this case. Hence the state-sum does not depend on the choice of the orientation of the 1 st sheet.

Suppose the 1st sheet is $-x+y+z=1$ as depicted in the top left of Figure 8 Then the figure shows that this case follows from the tetrahedral move with the 1st sheet $x+y+z=1$, together with creation/cancellation of a pair of triple points. Repeating this process, we conclude that the state-sum is independent of choice of the position of the 1 st sheet.

It remains to prove that the state-sum is independent of choice of the orientation of the 2nd sheet. In other words, we check the case where the sign of the triple 

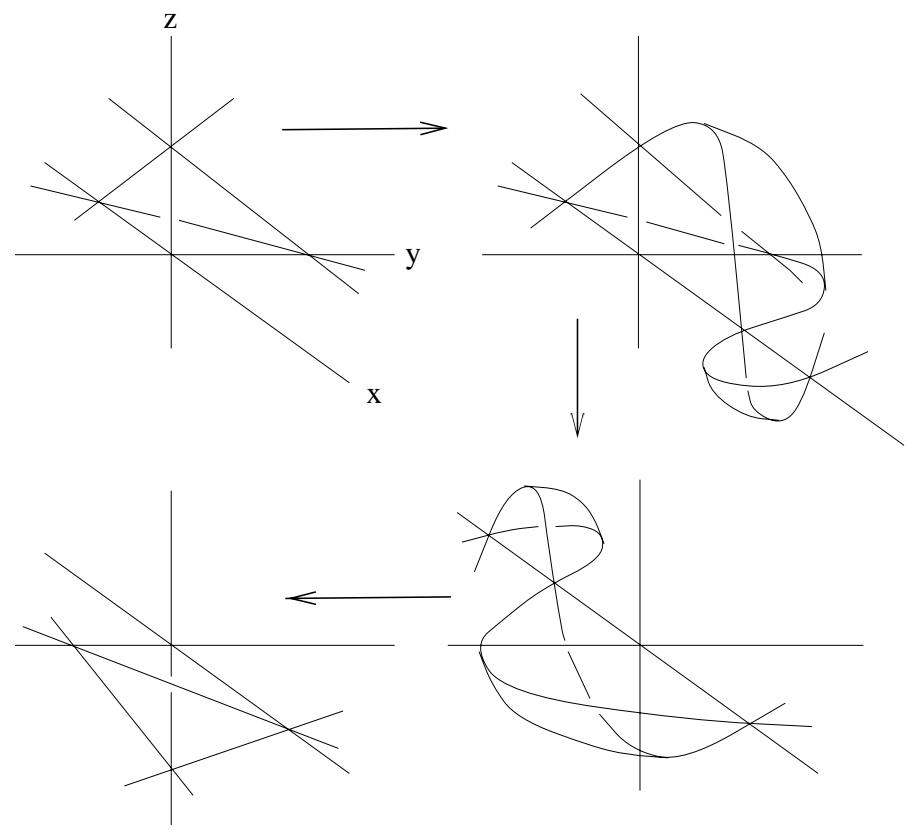

Figure 8. Turaev's technique generalized to four dimensions

point among the sheets $4,3,2$ is negative. In Figure 8, regard the $x y$-plane, $x z$ plane, and the plane $-x+y+z=1$ as the sheet 4,3 , and 2 respectively. Then the tetrahedral move from top right to the bottom right of the figure is the one with the opposite sign for the the sheets 4,3 , and 2, comparing to the tetrahedral move from top left to bottom left. Therefore this case also does not change the state-sum. This completes the proof.

5.7. Proposition. If $\Phi_{\theta}$ and $\Phi_{\theta^{\prime}}$ denote the state-sum invariants defined from cohomologous cocycles $\theta$ and $\theta^{\prime}$ (so that $\theta=\theta^{\prime} \delta \psi$ for some 2 -cochain $\psi$ ), then $\Phi_{\theta}=\Phi_{\theta^{\prime}}$ (so that $\Phi_{\theta}(K)=\Phi_{\theta^{\prime}}(K)$ for any knotted surface $\left.K\right)$.

In particular, the state-sum is equal to the number of colorings of a given knotted surface diagram if the 3-cocycle used for the Boltzmann weight is a coboundary.

Proof. We prove the second statement; the first follows a similar argument. Any orientable knotted surface can be isotoped in 4-dimensional space so that the projection has no branch points [17] (see also 8]). Thus we assume that the given projection does not have branch points. Then the double point set of the projection is a graph with 6 -valent vertices (that are triple points of the projection) possibly with loops with no vertices. Now the graph can be directed as follows [7. Give an orientation, specified by a vector $\vec{v}$, of an edge $e$ in such a way that $\vec{v}$ together with the normals $\overrightarrow{n_{1}}, \vec{n}_{2}$ of the top and bottom sheets of the projection (the triple $\left.\left(\vec{v}, \overrightarrow{n_{1}}, \overrightarrow{n_{2}}\right)\right)$ matches the orientation of the three-space. There are three edges going into the triple point and three coming out.

Suppose the $\theta$ is a coboundary. Then it is written as

$$
\theta(p, q, r)=\phi(p, r)^{-1} \phi(p, q) \phi(p * q, r) \phi(p * r, q * r)^{-1} \phi(q, r)^{-1} \phi(q, r)
$$


where the cancelling pair is added at the end of the RHS. The expression on the RHS consists of 2-cochains $\phi(x, y)$ where the pairs $(x, y)$ range over all pairs that appear at double curves near a triple point, when the triple point is colored in such a way that $\theta(p, q, r)^{ \pm 1}$ is its weight, where \pm 1 is the sign of the triple point. In other words, the cochain $\phi(x, y)$ corresponds to an edge with colors $x, y, x * y$. Furthermore, if an edge is oriented out of the triple point, then the cochain has negative exponent $\phi(x, y)$, and if the edge is oriented into the triple point, then the cochain has positive exponent. This is true for either sign $( \pm 1)$ of the triple point.

The weight $\theta(p, q, r)^{ \pm 1}$ may be considered to be the values $\phi(x, y)^{ \pm 1}$ assigned to the end points of six edges at the triple point. Note that if the edge comes out of the triple point, then the end point receives $\phi(x, y)^{-1}$, and if it comes in, then it receives $\phi(x, y)$. Now the state-sum for a fixed color is the product of all these weights assigned to end points of oriented edges. However, for every edge, the initial end point receives $\phi(x, y)^{-1}$ and the terminal point receives $\phi(x, y)$, so that the product of weights cancels out. Thus the product is 1 for any color, hence the state-sum is the number of colors of the diagram.

5.8. Remark. The fundamental quandle is defined (see 20 for example) for codimension 2 embeddings, and its presentations are defined (see [12] for example) using knot diagrams in all dimensions, along the line of Wirtinger presentations of knot groups. Let $Q(K)$ be the fundamental quandle of a knotted curve or surface $K$, and $X$ a finite quandle. Then it is seen using presentations of $Q(K)$ that there is a one-to-one correspondence between quandle homomorphisms $Q(K) \rightarrow X$ and colorings $\mathcal{C}: R \rightarrow X$.

\section{Computing QUANDle COHOMOLOGY}

In this section, quandle cohomology groups will be computed for some interesting examples. The cohomology groups will be computed directly from the definitions. More advanced techniques, such as exact sequences, would be desirable, but are not pursued here. We present some computational details, as some of the calculations will be used in later sections to find non-trivial invariants.

6.1. Remark. Suppose that the coefficient group $A$ is a cyclic group written additively as $\mathbf{Z}$ or $\mathbf{Z}_{n}$. Define a characteristic function

$$
\chi_{x}(y)= \begin{cases}1 & \text { if } x=y \\ 0 & \text { if } x \neq y\end{cases}
$$

from the free abelian group generated by $X^{n}$ to the group $A$.

The set $\left\{\chi_{x}: x \in X^{n}\right\}$ of such functions spans the group $C_{\mathrm{R}}^{n}(X ; A)$ of cochains. Thus if $f \in C_{\mathrm{R}}^{n}(X ; A)$ is a cochain, then

$$
f=\sum_{x \in X^{n}} C_{x} \chi_{x}
$$

If $f \in C_{\mathrm{Q}}^{n}(X ; A)$, then $f$ is written as

$$
f=\sum_{x \in X^{n} \backslash S} C_{x} \chi_{x}
$$


where $S=\left\{\left(x_{1}, \ldots, x_{n}\right): x_{j}=x_{j+1}\right.$ for some $\left.j=1, \ldots, n-1\right\}$. If $\delta f=0$, then $f$ vanishes on expressions of the form

$$
\sum_{j}(-1)^{j+1}\left(x_{0}, \ldots, \hat{x}_{j}, \ldots, x_{n}\right)+\sum_{k}(-1)^{k}\left(x_{0} * x_{k}, \ldots, x_{k-1} * x_{k}, x_{k+1}, \ldots, x_{n}\right) .
$$

In computing the cohomology we consider all such expressions as $\left(x_{0}, \ldots, x_{n}\right)$ ranges over all $(n+1)$-tuples for which each consecutive pair of elements is distinct. By evaluating linear combinations of characteristic functions on these expressions, we determine those functions that are cocycles. Similarly, we compute the coboundary on each of the characteristic functions in the previous dimension, to determine which linear combinations of characteristic functions are coboundaries. Since $A$ is a cyclic group, the generator will be denoted 1 (resp. $t$ ), the identity is denoted 0 (resp. 1), and the characteristic functions take values 0 or 1 (resp. 1 or $t$ ) when $A$ is written additively (resp. multiplicatively). We now turn to examples. All cohomology groups are quandle ones $H_{\mathrm{Q}}^{n}$, unless otherwise stated.

6.2. Definition ([12]). A rack is called trivial if $x * y=x$ for any $x, y$.

The dihedral quandle $R_{n}$ of order $n$ is the quandle consisting of reflections of the regular $n$-gon with the conjugation as operation. The dihedral group $D_{2 n}$ has a presentation

$$
\left\langle x, y \mid x^{2}=1=y^{n}, x y x=y^{-1}\right\rangle
$$

where $x$ is a reflection and $y$ is a rotation of a regular $n$-gon. The set of reflections $R_{n}$ in this presentation is $\left\{a_{i}=x y^{i}: i=0, \ldots, n-1\right\}$ where we use the subscripts from $\mathbf{Z}_{n}$ in the following computations. The operation is

$$
a_{i} * a_{j}=a_{j}^{-1} a_{i} a_{j}=x y^{j} x y^{i} x y^{j}=x y^{j} y^{-i} y^{j}=a_{2 j-i} .
$$

Hence $R_{n}$ is identified with $\mathbf{Z}_{n}=\{0, \ldots, n-1\}$, with quandle operation $i * j=2 j-i$ $(\bmod n)$. Compare with the well-known $n$-coloring of knot diagrams 15 .

Let $S_{4}$ denote the quandle with four elements, denoted by $0,1,2,3$, with the relations

$$
\begin{aligned}
& 0=0 * 0=1 * 2=2 * 3=3 * 1 \\
& 1=0 * 3=1 * 1=2 * 0=3 * 2, \\
& 2=0 * 1=1 * 3=2 * 2=3 * 0 \\
& 3=0 * 2=1 * 0=2 * 1=3 * 3
\end{aligned}
$$

This quandle is the set of clockwise rotations of the faces of a tetrahedron with conjugation as the operation.

6.3. Definition ([12, 27]). Let $\Lambda=\mathbf{Z}\left[T, T^{-1}\right]$ be the Laurent polynomial ring over the integers. Then any $\Lambda$-module $M$ has a quandle structure defined by $a * b=$ $T a+(1-T) b$ for $a, b \in M$.

For a Laurent polynomial $h(T)$ whose leading and terminal coefficients are \pm 1 , $\mathbf{Z}_{n}\left[T, T^{-1}\right] /(h(T))$ is a finite quandle. We call such quandles $(\bmod n)$-Alexander quandles. Alexander quandles are of interest in Section 8

6.4. Proposition. We have the following two quandle isomorphisms:

$$
R_{4} \cong \mathbf{Z}_{2}\left[T, T^{-1}\right] /\left(T^{2}-1\right) \text { and } S_{4} \cong \mathbf{Z}_{2}\left[T, T^{-1}\right] /\left(T^{2}+T+1\right) .
$$

Proof. The set of elements of either of these Alexander quandles can be represented as $\{0,1, T, 1+T\}$. The following assignment defines an isomorphism $R_{4} \cong \mathbf{Z}_{2}\left[T, T^{-1}\right] /\left(T^{2}-1\right): 0 \leftrightarrow 0,1 \leftrightarrow 1,2 \leftrightarrow 1+T$, and $3 \leftrightarrow T$. It happens that the same correspondence also gives an isomorphism to $S_{4}$. 
6.5. Lemma. Any cochain on a trivial quandle is a cocycle. Only the zero map is a coboundary.

Proof. This follows from the definitions.

It is worth remarking here that the trivial quandle is quite effective in detecting linking. See Section 8

6.6. Lemma. $H^{2}\left(R_{3} ; \mathbf{Z}\right) \cong 0$.

Proof. Let a 2-cocycle $f \in Z^{2}\left(R_{3} ; \mathbf{Z}\right)$ be expressed as

$$
f=\sum_{i, j \in R_{3}} C_{(i, j)} \chi_{(i, j)} .
$$

Then

and

$$
C_{(p, r)}+C_{(p * r, q * r)}-C_{(p, q)}-C_{(p * q, r)}=0 \quad \text { for } p, q, r \in R_{3}
$$

$$
C_{(p, p)}=0 \quad \text { for } p \in R_{3} .
$$

The quandle $R_{3}$ has three elements, $0,1,2$ with quandle operation

$$
i * j=2 j-i \quad(\bmod 3) .
$$

Substituting 0,1,2 for all possibilities for the variables $p, q, r$ into the above expressions, we have 30 equations on $C_{(i, j)}$, which are simplified as the following:

$$
\begin{aligned}
C_{(0,1)}+C_{(2,1)} & =0 \\
C_{(0,2)}-C_{(2,0)}+C_{(2,1)} & =0 \\
C_{(1,0)}+C_{(2,0)} & =0 \\
C_{(0,2)}+C_{(1,2)} & =0 \\
C_{(i, i)} & =0 \text { for } i \in\{0,1,2\} .
\end{aligned}
$$

Therefore,

$$
\begin{array}{lll}
C_{(0,0)}=0, & C_{(0,1)}=\alpha, & C_{(0,2)}=\beta, \\
C_{(1,0)}=\alpha-\beta, & C_{(1,1)}=0, & C_{(1,2)}=-\beta, \\
C_{(2,0)}=\beta-\alpha, & C_{(2,1)}=-\alpha, & C_{(2,2)}=0,
\end{array}
$$

where we put $C_{(0,1)}=\alpha$ and $C_{(0,2)}=\beta$. Then

$$
f=\alpha\left[\chi_{(0,1)}+\chi_{(1,0)}-\chi_{(2,0)}-\chi_{(2,1)}\right]+\beta\left[\chi_{(0,2)}-\chi_{(1,0)}-\chi_{(1,2)}+\chi_{(2,0)}\right] .
$$

Since

$$
\begin{aligned}
& \delta \chi_{1}=-\chi_{(0,2)}+\chi_{(1,0)}+\chi_{(1,2)}-\chi_{(2,0)}, \\
& \delta \chi_{2}=-\chi_{(0,1)}-\chi_{(1,0)}+\chi_{(2,0)}+\chi_{(2,1)},
\end{aligned}
$$

we see that $f$ is a coboundary.

6.7. Lemma. $H^{2}\left(R_{4} ; \mathbf{Z}\right) \cong \mathbf{Z} \times \mathbf{Z}$.

Proof. The quandle $R_{4}$ has four elements, 0,1,2, and 3; and the quandle operation is

$$
i * j=2 j-i \quad(\bmod 4) .
$$

Let a 2-cocycle $f \in Z^{2}\left(R_{4} ; \mathbf{Z}\right)$ be expressed as

$$
f=\sum_{i, j \in R_{4}} C_{(i, j)} \chi_{(i, j)} .
$$


Then

$$
\begin{aligned}
C_{(0,1)}-C_{(0,3)}+C_{(2,1)}-C_{(2,3)} & =0, \\
C_{(0,2)}+C_{(2,1)}-C_{(2,3)} & =0, \\
C_{(1,3)}+C_{(3,1)} & =0, \\
C_{(2,0)}+C_{(2,1)}-C_{(2,3)} & =0, \\
-C_{(2,0)}+C_{(2,1)}-C_{(2,3)} & =0, \\
C_{(1,0)}-C_{(1,2)}+C_{(3,1)} & =0, \\
-C_{(1,3)}+C_{(3,1)} & =0, \\
C_{(3,0)}+C_{(3,1)}-C_{(3,2)} & =0, \\
C_{(i, i)} & =0 \text { for } i \in\{0,1,2,3\} .
\end{aligned}
$$

Thus

$$
\begin{aligned}
& C_{(1,3)}=C_{(3,1)}, \\
& C_{(0,2)}=C_{(2,0)},
\end{aligned}
$$

and

$$
2 C_{(1,3)}=2 C_{(0,2)}=0 .
$$

The relations among the coefficients give that the group of 2-cocycles is represented as

$$
Z^{2}\left(R_{4} ; \mathbf{Z}\right) \cong \operatorname{Hom}\left(\mathbf{Z}^{4} \times\left(\mathbf{Z}_{2}\right)^{2}, \mathbf{Z}\right) \cong \mathbf{Z}^{4}
$$

where the generators are

$$
\begin{aligned}
& f_{(0,1)}=\chi_{(0,1)}+\chi_{(0,3)}, \\
& f_{(2,1)}=\chi_{(2,1)}+\chi_{(2,3)}, \\
& f_{(1,0)}=\chi_{(1,0)}+\chi_{(1,2)}, \\
& f_{(3,0)}=\chi_{(3,0)}+\chi_{(3,2)} .
\end{aligned}
$$

The coboundaries are computed as follows:

$$
\begin{aligned}
\delta \chi_{0} & =\chi_{(0,1)}+\chi_{(0,3)}-\chi_{(2,1)}-\chi_{(2,3)} \\
& =f_{(0,1)}-f_{(2,1)}, \\
\delta \chi_{2} & =-\chi_{(0,1)}-\chi_{(0,3)}+\chi_{(2,1)}+\chi_{(2,3)} \\
& =-f_{(0,1)}+f_{(2,1)}, \\
\delta \chi_{1} & =\chi_{(1,0)}+\chi_{(1,2)}-\chi_{(3,0)}-\chi_{(3,2)} \\
\delta \chi_{3} & =-\chi_{(1,0)}-f_{(3,0)}, \\
& =-f_{(1,0)}-\chi_{(1,2)}+\chi_{(3,0)} .
\end{aligned}
$$

Therefore $H^{2}\left(R_{4} ; \mathbf{Z}\right) \cong \mathbf{Z}^{2}$.

We have the following calculations that were performed using Mathematica and Maple. (See also 4, 18] for more on the 2nd homology of the dihedral quandles.)

6.8. Lemma. For the 3-element dihedral quandle we have

$$
H^{3}\left(R_{3} ; \mathbf{Z}_{3}\right) \cong \mathbf{Z}_{3} \quad \text { and } \quad H^{3}\left(R_{3} ; \mathbf{Z}\right) \cong 0 \text {. }
$$


Proof. We summarize the calculation. For any coefficient group, any cocycle can be written as $\sum_{i=1}^{5} a_{i} \eta_{i}$ where $3 a_{1}=0$, and

$$
\begin{aligned}
& \eta_{1}=-\chi_{(0,1,0)}+\chi_{(0,2,0)}+2 \chi_{(0,2,1)}+\chi_{(1,0,1)}+\chi_{(1,0,2)}+\chi_{(2,0,2)}+\chi_{(2,1,2)} \\
& \eta_{2}=-\chi_{(0,1,0)}+\chi_{(0,2,1)}-\chi_{(1,0,1)}+\chi_{(1,2,0)} \\
& \eta_{3}=\chi_{(0,1,0)}+\chi_{(0,1,2)}-\chi_{(0,2,0)}-\chi_{(0,2,1)}-\chi_{(1,0,2)}+\chi_{(1,2,1)} \\
& \eta_{4}=\chi_{(0,1,0)}+\chi_{(0,1,2)}-\chi_{(0,2,0)}-\chi_{(0,2,1)}+\chi_{(2,0,1)}-\chi_{(2,1,2)} \\
& \eta_{5}=\chi_{(0,1,2)}-\chi_{(0,2,0)}-\chi_{(2,0,2)}+\chi_{(2,1,0)}
\end{aligned}
$$

For example, if the coefficient group is $\mathbf{Z}$, then $Z^{3}\left(R_{3} ; \mathbf{Z}\right)=\mathbf{Z}^{4}$ and is generated by $\eta_{2}, \eta_{3}, \eta_{4}$, and $\eta_{5}$. If the coefficient group is $\mathbf{Z}_{3}$, then $Z^{3}\left(R_{3} ; \mathbf{Z}_{3}\right)=\left(\mathbf{Z}_{3}\right)^{5}$ and is generated by $\eta_{1}, \eta_{2}, \eta_{3}, \eta_{4}$, and $\eta_{5}$.

The following elements generate the group of coboundaries:

$$
\begin{aligned}
& \delta \chi_{(0,1)}=-\chi_{(0,1,0)}-\chi_{(0,1,2)}+\chi_{(0,2,0)}+\chi_{(0,2,1)}+\chi_{(1,0,2)}-\chi_{(1,2,1)} \\
& \delta \chi_{(0,2)}=\chi_{(0,1,0)}+\chi_{(0,1,2)}-\chi_{(0,2,0)}-\chi_{(0,2,1)}+\chi_{(2,0,1)}-\chi_{(2,1,2)} \\
& \delta \chi_{(1,0)}=\chi_{(0,1,2)}-\chi_{(0,2,0)}-\chi_{(1,0,1)}-\chi_{(1,0,2)}+\chi_{(1,2,0)}+\chi_{(1,2,1)} \\
& \delta \chi_{(1,2)}=\chi_{(1,0,1)}+\chi_{(1,0,2)}-\chi_{(1,2,0)}-\chi_{(1,2,1)}-\chi_{(2,0,2)}+\chi_{(2,1,0)} \\
& \delta \chi_{(2,0)}=-\chi_{(0,1,0)}+\chi_{(0,2,1)}-\chi_{(2,0,1)}-\chi_{(2,0,2)}+\chi_{(2,1,0)}+\chi_{(2,1,2)} \\
& \delta \chi_{(2,1)}=-\chi_{(1,0,1)}+\chi_{(1,2,0)}+\chi_{(2,0,1)}+\chi_{(2,0,2)}-\chi_{(2,1,0)}-\chi_{(2,1,2)}
\end{aligned}
$$

Comparing the cocycles and coboundaries, we have the result.

6.9. Remark. In Section [11, we use the 3-cocycle

$\eta_{1}=-\chi_{(0,1,0)}+\chi_{(0,2,0)}-\chi_{(0,2,1)}+\chi_{(1,0,1)}+\chi_{(1,0,2)}+\chi_{(2,0,2)}+\chi_{(2,1,2)} \in Z^{3}\left(R_{3} ; \mathbf{Z}_{3}\right)$

to distinguish the 2-twist spun trefoil from its orientation reversed image.

6.10. Remark. Similar computations give the following results that are used to compute knot invariants in a subsequent paper:

$$
\begin{gathered}
H^{2}\left(S_{4} ; A\right)= \begin{cases}\mathbf{Z}_{2} & \text { for } A=\mathbf{Z}_{2}, \\
0 & \text { for } A=\mathbf{Z} ;\end{cases} \\
H^{3}\left(S_{4} ; A\right)= \begin{cases}\mathbf{Z}_{2} & \text { for } A=\mathbf{Z}, \\
0 & \text { for } A=\mathbf{Q}, \\
\left(\mathbf{Z}_{2}\right)^{3} & \text { for } A=\mathbf{Z}_{2}, \\
\left(\mathbf{Z}_{2}\right)^{2} \times \mathbf{Z}_{4} & \text { for } A=\mathbf{Z}_{4} .\end{cases}
\end{gathered}
$$

\section{Group 2-COCyCles AND QUANDle 2-COCyCles}

In this section we give quandle 2-cocycles using group 2-cocycles. Let $G$ be a group and let $A$ be an abelian group (written multiplicatively) upon which the group ring $\mathbf{Z}[G]$ acts trivially. Then the group cohomology is defined from the following cochain complex. The abelian group of all maps from the cartesian product of $n$ 


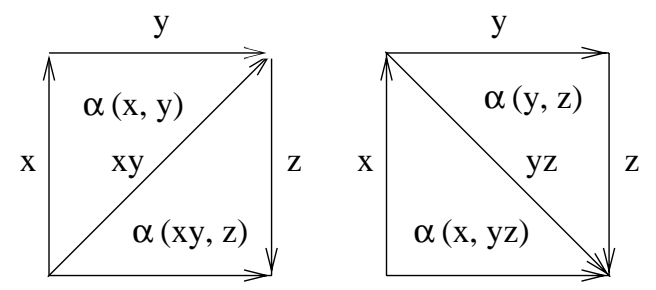

FIGURE 9. Group 2-cocycle condition and triangulations of a square

copies of $G$ to $A$ is denoted by $C^{n}(G ; A)$. A coboundary operator $\delta: C^{n}(G ; A) \rightarrow$ $C^{n+1}(G ; A)$ is defined by

$$
\begin{aligned}
& (\delta f)\left(x_{1}, \ldots, x_{n+1}\right) \\
& \quad=f\left(x_{2}, \ldots, x_{n+1}\right) \prod_{i=1}^{n} f\left(x_{1}, \ldots, x_{i} x_{i+1}, \ldots, x_{n+1}\right)^{(-1)^{i}} f\left(x_{1}, \ldots, x_{n}\right)^{(-1)^{n+1}},
\end{aligned}
$$

where $f \in C^{n}(G ; A)$ and $x_{1}, \ldots, x_{n+1} \in G$.

In particular, a function $\alpha: G \times G \rightarrow A$ satisfies the group 2-cocycle condition if the following relation holds:

$$
\alpha(x, y) \alpha(x y, z)=\alpha(x, y z) \alpha(y, z)
$$

The diagrammatic interpretation of this condition is depicted in Figure 9. Consider triangulations of planar regions. Suppose the edges are oriented in such a way that at every triangle, exactly two edges point to the same orientation (clockwise or counterclockwise) and one edge has the opposite direction. Let $G$ be a finite group, and assign elements of $G$ on the edges, such that if the two edges of the same directions receive $x$ and $y$ in this direction, then the other edge receives $x y$. The value $\alpha(x, y)$ of a 2-cocycle $\alpha$ is assigned to such a triangle [9, 16] (see also [5]). With this convention, two ways of triangulating a square correspond to the 2-cocycle condition as depicted in Figure 9 .

7.1. Theorem. Let $G$ be a group, considered also as a quandle by conjugation that we denote by $G_{\text {conj. }}$. Let $\alpha \in Z^{2}(G ; A)$ be a group 2-cocycle. Define a quandle 2-cochain

$$
\phi(p, q)=\alpha(p, q) \alpha\left(q, q^{-1} p q\right)^{-1}
$$

Then $\phi$ is a quandle 2 -cocycle, $\phi \in Z^{2}\left(G_{\mathrm{conj}} ; A\right)$.

Proof. A similar argument to that in [50] shows that a group 2-cocycle $\alpha$ satisfies

$$
\begin{aligned}
& \alpha(x, y)=\alpha\left(x^{-1}, x y\right)^{-1}=\alpha\left(x y, y^{-1}\right)^{-1} \\
& \alpha(x, y)=\alpha\left(z, z^{-1} x\right)^{-1} \alpha\left(z^{-1} x, y\right) \alpha\left(z, z^{-1} x y\right) .
\end{aligned}
$$



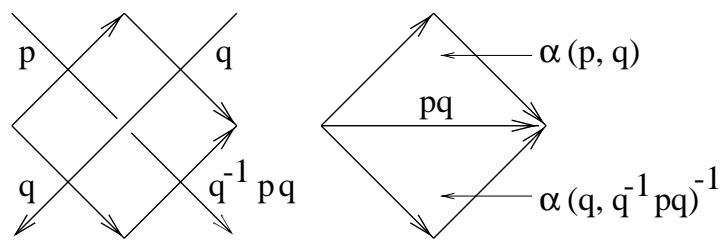

Figure 10. Defining a quandle cocycle from a group cocycle

Using these identities and 2-cocycle conditions, one computes

$$
\begin{aligned}
& \phi(p, q) \phi(p * q, r) \phi(q, r) \\
& =\alpha(p, q) \alpha\left(q, q^{-1} p q\right)^{-1} \alpha\left(q^{-1} p q, r\right) \alpha\left(r, r^{-1} q^{-1} p q r\right)^{-1} \alpha(q, r) \alpha\left(r, r^{-1} q r\right)^{-1} \\
& =\alpha(p, q) \underline{\alpha\left(q, q^{-1} p q\right)^{-1} \alpha\left(q^{-1} p q, q^{-1} p^{-1} q r\right)} \\
& \times \alpha\left(q^{-1} p^{-1} q r, r^{-1} q^{-1} p q r\right)^{-1} \alpha(q, r) \alpha\left(r, r^{-1} q r\right)^{-1} \\
& =\alpha(p, q) \alpha\left(p q, q^{-1} p^{-1} q r\right) \alpha\left(q^{-1} p^{-1} q r, r^{-1} q^{-1} p q r\right)^{-1} \alpha\left(r, r^{-1} q r\right)^{-1} \\
& \left.=\frac{\alpha(p, q) \alpha\left(p r, r^{-1} q\right)^{-1} \alpha}{} \alpha r^{-1} q, q^{-1} p^{-1} q r\right) \alpha\left(p r, r^{-1} p^{-1} q r\right) \\
& \times \alpha\left(q^{-1} p^{-1} q r, r^{-1} q^{-1} p q r\right)^{-1} \alpha\left(r, r^{-1} q r\right)^{-1} \\
& =\alpha(p, r) \alpha\left(r, r^{-1} q\right)^{-1} \alpha\left(r^{-1} q, q^{-1} p^{-1} q r\right) \alpha\left(p r, r^{-1} p^{-1} q r\right) \\
& \times \alpha\left(q^{-1} p^{-1} q r, r^{-1} q^{-1} p q r\right)^{-1} \alpha\left(r, r^{-1} q r\right)^{-1} \\
& =\alpha(p, r) \alpha\left(r, r^{-1} q\right)^{-1} \alpha\left(r^{-1} q, q^{-1} p^{-1} q r\right) \alpha\left(r, r^{-1} p r\right)^{-1} \\
& \times \alpha\left(r^{-1} p r, r^{-1} p^{-1} q r\right) \alpha\left(q^{-1} p^{-1} q r, r^{-1} q^{-1} p q r\right)^{-1} \\
& =\alpha(p, r) \alpha\left(r, r^{-1} q\right)^{-1} \alpha\left(r^{-1} q, r\right) \alpha\left(r^{-1} p^{-1} q r, r^{-1} q^{-1} p q r\right)^{-1} \\
& \times \alpha\left(r, r^{-1} p r\right)^{-1} \alpha\left(r^{-1} p r, r^{-1} p^{-1} q r\right) \\
& =\alpha(p, r) \alpha(q, r) \alpha\left(r, r^{-1} q r\right)^{-1} \alpha\left(r^{-1} p^{-1} q r, r^{-1} q^{-1} p q r\right)^{-1} \\
& \times \alpha\left(r, r^{-1} p r\right)^{-1} \underline{\alpha\left(r^{-1} p r, r^{-1} p^{-1} q r\right)} \\
& =\alpha(p, r) \alpha(q, r) \alpha \overline{\left(r, r^{-1} q r\right)^{-1} \alpha\left(r^{-1} p r, r^{-1} q r\right)} \\
& \times \alpha\left(r^{-1} q r, r^{-1} p^{-1} q r\right)^{-1} \alpha\left(r, r^{-1} p r\right)^{-1} \\
& =\phi(q, r) \phi(p, r) \phi(p * r, q * r) .
\end{aligned}
$$

The above computation is easily carried out using diagrams. At a crossing, a square is assigned as in Figure 10 left. Then the square is triangulated, and group cocycles are assigned as in Figure 9 The Reidemeister type III move is then interpreted as changes of triangulations of squares, giving the above computations.

\section{COMPUTATIONS OF COCYCLE INVARIANTS OF ClASSiCAL KNOTS AND LINKS}

Suppose a link, $L$, is colored by the trivial $n$-element quandle, $T_{n}$, whose elements we represent by integers $T_{n}=\{1, \ldots, n\}$. Since $a * b=a$ for all $a$ and $b \in T_{n}$, each component of a link $L$ is monochromatically colored. More precisely, if $a_{i}$, $i=1, \ldots, m$, are $\operatorname{arcs}$ of a component $K$ of $L$, the color $\mathcal{C}\left(a_{i}\right)$ takes the same value in $T_{n}$ for $i=1, \ldots, m$. We assume that the coefficient group $A$ is cyclic and generated by $t$ (which is infinite cyclic for a while). The coboundary homomorphism $\delta$ is trivial 
for $T_{n}$ for any $n$, and in particular, any function $\phi$ is a cocycle in $T_{n}$. Consider the characteristic functions (that we write multiplicatively for this section):

$$
\chi_{(x, y)}(a, b)= \begin{cases}t & \text { if }(a, b)=(x, y), \\ 1 & \text { otherwise. }\end{cases}
$$

For an $n$-component link $L=K_{1} \cup \ldots \cup K_{n}$, let $\ell k\left(K_{i}, K_{j}\right)$ denote the linking number of the pair $\left(K_{i}, K_{j}\right)$ of components, and let $\ell k(L)=\sum_{i<j} \ell k\left(K_{i}, K_{j}\right)$ denote the total linking number, where the sum ranges over all pairs with $i<j, i, j=$ $1, \ldots, n$. Define also the linking number $\ell k(A, B)$ for any disjoint pair of subsets $A, B \subset\left\{K_{1}, \ldots, K_{n}\right\}$ by $\ell k(A, B)=\sum_{K_{u} \in A, K_{v} \in B} \ell k\left(K_{u}, K_{v}\right)$, where $\ell k(A, \emptyset)=$ $0=\ell k(\emptyset, B)$. Recall that the linking number of a 2-component classical link $L=$ $K_{1} \cup K_{2}$ can be computed by counting the crossing number with signs $( \pm 1)$ where the component $K_{1}$ crosses over $K_{2}$ 38.

8.1. Theorem. For any cocycle of $T_{k}$, where $k$ is any positive integer, and for any link $L$, the state-sum $\Phi(L)$ is a function of pairwise linking numbers.

Proof. Let the elements of $T_{k}$ be denoted by $1, \ldots, k$, and let $\phi=\prod_{i \neq j} \chi_{(i, j)}^{w_{i, j}} \in$ $Z^{2}\left(T_{k} ; \mathbf{Z}\right.$ ) (any cocycle can be written this way for some integers $w_{i, j}$ ). For each coloring of $L$ by $T_{k}$, there is an ordered partition $A=\left\{A_{1}, \ldots, A_{k}\right\}$ of $\left\{K_{1}, \ldots, K_{n}\right\}$ such that each component of $A_{j}$ is colored by $j \in T_{k}$ where $L=K_{1} \cup \ldots \cup K_{n}$. All ordered partitions of $\left\{K_{1}, \ldots, K_{n}\right\}$ are in one-to-one correspondence to colorings by $T_{k}$. Then the state-sum invariant $\Phi(L)$ with respect to this cocycle is written as

$$
\sum_{A} \prod_{i, j} t^{\ell k\left(A_{i}, A_{j}\right) w_{i, j}}
$$

where $A$ ranges over all ordered partitions of components.

In particular, for $T_{2}$ and for links with small numbers of components, we obtain the following formulas by counting componentwise crossing numbers.

8.2. Proposition. Take $\phi=\chi_{(1,2)} \in Z^{2}\left(T_{2} ; \mathbf{Z}\right)$ to define a cocycle invariant $\Phi(L)=$ $\Phi_{\phi}(L)$ for a link (or a knot) $L$.

(1) If $K$ is a knot, then $\Phi(K)=2$.

(2) If $L=K_{1} \cup K_{2}$ is a 2-component link, then

$$
\Phi(L)=2\left(1+t^{\ell k(L)}\right) .
$$

(3) If $L=K_{1} \cup K_{2} \cup K_{3}$ is a 3-component link, then

$$
\Phi(L)=2\left(1+\sum_{i, j=1, i<j}^{3} t^{\ell k(L)-\ell k\left(K_{i}, K_{j}\right)}\right) .
$$

Next we study invariants with dihedral quandles. We consider the dihedral quandle of four elements as $R_{4}=\left\{a_{1}, a_{2}, b_{1}, b_{2}: a_{i} * a_{j}=a_{i}, b_{i} * b_{j}=b_{i}, a_{i} * b_{j}=\right.$ $\left.a_{i+1}, b_{i} * a_{j}=b_{i+1}\right\}$ where, in the subscripts, $2+1$ is taken to be 1 . Geometrically $a_{1}, a_{2}, b_{1}$, and $b_{2}$ represent the reflections of a square about the horizontal axis, vertical axis, the line $y=x$, the line $y=-x$, respectively.

8.3. Example $((4,2)$-torus link). A computation of the state-sum invariant for the $(4,2)$-torus link with $X=R_{4}$ is depicted in Figure 11 We assume $A=\mathbf{Z}=\langle t\rangle$. 


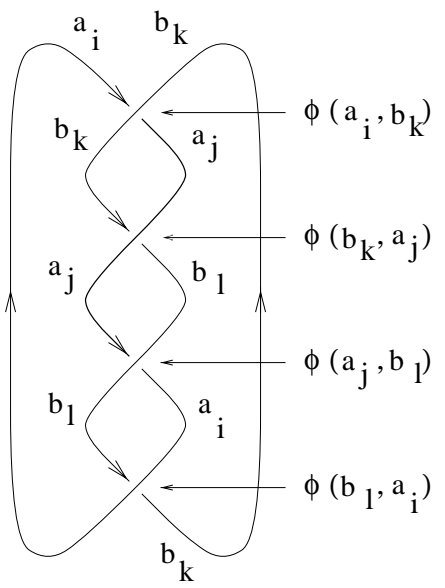

FiguRE 11. Computations for $(4,2)$-torus link

First, we consider a 2-cocycle $\phi=f_{\left(a_{1}, b_{1}\right)}=\chi_{\left(a_{1}, b_{1}\right)} \chi_{\left(a_{1}, b_{2}\right)}$ (in multiplicative notation). In Figure 11 a specific coloring and the corresponding weights are shown. All possible colorings are obtained as follows. If only one quandle element is used, the coloring's state-sum contribution is trivial (1). Since $R_{4}$ has 4 elements, there are 4 such possibilities. If one component is colored by $a_{1}$, and the other by $a_{2}$, there are no crossings of weight $\phi\left(a_{1}, b_{j}\right)^{ \pm 1}$ for $j \in\{1,2\}$, so these two colorings give trivial state-sum contributions as well. Coloring one component by $b_{1}$ and the other by $b_{2}$ produces 2 similar cases. When one component is colored by the $a$ 's and the other by the $b$ 's, the color contributes $t$ to the state-sum. There are 8 such colorings, one of which is depicted in Figure 11 Since these cases cover all possible colorings, the state-sum is $8+8 t=8(1+t)$.

In general we have the following lemma on colorings by $R_{4}$, which is proved by induction on the number of components $n$.

8.4. Lemma. Let $L=K_{1} \cup \ldots \cup K_{n}$ be a link such that any pairwise linking number is even. Then for any arcs $r_{i}$ of $K_{i}, i=1, \ldots, n$, and for any color on $r_{i}$ $(i=1, \ldots, n)$ by $R_{4}$, there is a unique coloring of $L$ that extends the given coloring on $r_{i}(i=1, \ldots, n)$. In particular, the number of colorings is $4^{n}$.

From the proof of Lemma 6.7 any cohomology class in $H^{2}\left(R_{4} ; \mathbf{Z}\right)$ is represented by a cocycle of the form $\phi=\lambda_{1}^{u} \lambda_{2}^{v}$ for some integers $u$ and $v$ where $\lambda_{1}=\chi_{\left(a_{1}, b_{1}\right)} \chi_{\left(a_{1}, b_{2}\right)}$ and $\lambda_{2}=\chi_{\left(a_{2}, b_{1}\right)} \chi_{\left(a_{2}, b_{2}\right)}$. Hence by Lemma 4.5 all possible values of the state-sum invariant with $R_{4}$ can be obtained by examining the cocycles of the above form.

8.5. Theorem. The state-sum invariant $\Phi(L)$ with respect to the cocycle $\phi=\lambda_{1}^{u} \lambda_{2}^{v}$ of $R_{4}$, of any $n$-component link $L=K_{1} \cup \ldots \cup K_{n}$ such that any pairwise linking number is even, is of the form

$$
\Phi(L)=2^{n}\left(\sum_{A \in \mathcal{P}(\mathcal{K})} t^{(u+v) \ell k(A, B) / 2}\right)
$$

where $\mathcal{K}=\left\{K_{1}, \ldots, K_{n}\right\}, \mathcal{P}(\mathcal{K})$ denotes its power set, and $B=\mathcal{K} \backslash A$. 
For example, for 1-, 2-, 3-component links $L$, respectively,

$$
\begin{aligned}
& \Phi(L)=4, \\
& \Phi(L)=8\left(1+t^{(u+v) \ell k(L) / 2}\right) . \\
& \Phi(L)=16\left(1+\sum_{i, j=1, i<j}^{3} t^{(u+v)\left(\ell k(L)-\ell k\left(K_{i}, K_{j}\right) / 2\right)}\right) .
\end{aligned}
$$

Proof. Write $L=(\bigcup A) \cup(\cup B)$ and take a color that assigns $a_{i}$ 's to $A$ and $b_{j}$ 's to $B$. Let $Y_{i}^{ \pm}$be the number of crossings of a diagram of $L$ of sign \pm where the arc colored $a_{i}$ goes under an arc colored by $b_{1}$ or $b_{2}$ and comes out with the color $a_{i+1}$. Then with $\phi$, the state-sum contribution of this color is $t^{u\left(Y_{1}^{+}-Y_{2}^{-}\right)+v\left(Y_{2}^{+}-Y_{1}^{-}\right)}$. When we trace each component $K_{i}$ of $A$, the colors alternate $a_{1}$ and $a_{2}$ at the crossings of the above types. Therefore

$$
Y_{1}^{+}+Y_{1}^{-}=Y_{2}^{+}+Y_{2}^{-}
$$

which is equivalent to

$$
Y_{1}^{+}-Y_{2}^{-}=Y_{2}^{+}-Y_{1}^{-},
$$

so the contribution is written as

$$
t^{u\left(Y_{1}^{+}-Y_{2}^{-}\right)+v\left(Y_{2}^{+}-Y_{1}^{-}\right)}=t^{(u+v)\left(Y_{1}^{+}-Y_{2}^{-}\right)} .
$$

On the other hand, one computes

$$
\begin{aligned}
\ell k(L) & =\left(Y_{1}^{+}+Y_{2}^{+}\right)-\left(Y_{1}^{-}+Y_{2}^{-}\right) \\
& =2\left(Y_{1}^{+}-Y_{2}^{-}\right)
\end{aligned}
$$

and the result follows. From the uniqueness in Lemma 8.4 the number of such colorings is (the number of colorings of components in $A$ by $a_{i}{ }^{\prime}$ 's) $\times$ (the number of colorings of components in $B$ by $\left.b_{j}{ }^{\prime} \mathrm{s}\right)=2^{|A|} \times 2^{|B|}=2^{n}$.

Recall that a map $f: X \rightarrow Y$ between two quandles $X, Y$ is called a (quandle) homomorphism if $f(a * b)=f(a) * f(b)$ for any $a, b \in X$. A homomorphism is called an isomorphism if it is bijective. An isomorphism $f: X \rightarrow X$ is called an automorphism (see [20]).

Next we consider invariants with general dihedral quandles. For a dihedral quandle $R_{n}$, use integers modulo $n, R_{n}=\{0,1, \ldots, n-1\}$, with the quandle operation $i * j=2 j-i(\bmod n)$. Denote by $R_{2 n}^{0}$ (respectively $\left.R_{2 n}^{1}\right)$ the evens (resp. odds) of $R_{2 n}$. It is obvious that for any coloring of a link of $L$ by $R_{2 n}$, each component of $L$ is colored either by $R_{2 n}^{0}$ or by $R_{2 n}^{1}$.

8.6. Lemma. If a knot $K$ has a non-trivial state-sum with $R_{2 n}$ associated with a 2 -cocycle $\phi \in C^{2}\left(R_{2 n} ; \mathbf{Z}\right)$, then there is a 2 -cocycle $\phi^{\prime} \in C^{2}\left(R_{n} ; \mathbf{Z}\right)$ such that $K$ has a non-trivial state-sum with $R_{n}$ associated with $\phi^{\prime}$.

Proof. Let $\mathcal{C}$ be a coloring such that $\phi$ applied to the coloring $\mathcal{C}$ of $K$ produces $t^{q}$-terms for some $q \in \mathbf{Z}-0$; i.e.,

$$
\prod_{\tau} B(\tau, \mathcal{C})=t^{q} \quad \text { for some } q \in \mathbf{Z}-0 .
$$

Since $K$ is a knot, all colors used in $\mathcal{C}$ are either elements of $R_{2 n}^{1}$ or elements of $R_{2 n}^{0}$. For the first case, consider an isomorphism $j_{1}: R_{2 n}^{1} \rightarrow R_{n}, x \mapsto \frac{x-1}{2}$. (The inverse is $x \mapsto 2 x+1$.) The cocycle $\phi$ induces a cocycle $\phi^{\prime} \in C^{2}\left(R_{n} ; \mathbf{Z}\right)$ by 
$\phi^{\prime}(x, y)=\phi\left(j_{1}^{-1}(x), j_{1}^{-1}(y)\right)$. Note that $\phi^{\prime}$ has the same value on the color $j_{1}(\mathcal{C})$ as $\phi$ did on $\mathcal{C}$. Thus, $K$ has a non-trivial state-sum with $R_{n}$ associated with $\phi^{\prime}$. The other case is proved similarly by use of $j_{0}: R_{2 n}^{0} \rightarrow R_{n}, x \mapsto \frac{x}{2}$ (and the inverse $x \mapsto 2 x)$.

8.7. Corollary. All knots have trivial state-sums with any dihedral quandle $R_{n}$, $n \geq 2$, associated with any 2 -cocycle $\phi \in C^{2}\left(R_{n} ; \mathbf{Z}\right)$.

Proof. First note that $R_{2}$ is isomorphic to $T_{2}$, the trivial two element quandle. Then all knots in $R_{2}$ have trivial state-sum. Then, by induction using the above lemma, we see that any knot has the trivial state-sum with $R_{2^{m}}$ for all $m \geq 1$. The dihedral quandles with an odd number of elements have no 2-dimensional integral quandle cohomology [18, 4]. Hence the same argument, using the above lemma, gives the result.

Next we consider invariants with Alexander quandles.

8.8. Lemma. Let $n$ be a positive integer $(>1)$ and $h(T) \in \mathbf{Z}_{n}\left[T, T^{-1}\right]$.

(a) If $n \mid h(1)$, then the map $p: \mathbf{Z}_{n}\left[T, T^{-1}\right] /(h(T)) \rightarrow \mathbf{Z}_{n}$ defined by $f(T) \mapsto f(1)$ defines a surjective homomorphism to the trivial quandle. Here $\mathbf{Z}_{n}$ is given the quandle structure of $T_{n}$.

(b) If $n \mid h(-1)$, then the map $q: \mathbf{Z}_{n}\left[T, T^{-1}\right] /(h(T)) \rightarrow \mathbf{Z}_{n}$ defined by $f(T) \mapsto$ $f(-1)$ defines a surjective homomorphism to the dihedral quandle. Here $\mathbf{Z}_{n}$ is given the quandle structure of $R_{n}$.

Proof. The operation $a * b=T a+(1-T) b$ on Alexander quandles become $a * b=a$ for $T=1$ and $a * b=2 b-a$ for $T=-1$.

8.9. Remark. (1) Observe that part (b) corresponds to the existence of Fox colorings if and only if $n$ divides the determinant of the knot.

(2) In the above lemma (a), let $\phi_{i, j} \in Z^{2}\left(\mathbf{Z}_{n}\left[T, T^{-1}\right] /(h(T)) ; \mathbf{Z}\right)$ be the pull-back cocycle $p^{\sharp} \chi_{(i, j)}$ by the homomorphism $p$ of a cocycle $\chi_{(i, j)} \in Z^{2}\left(T_{n} ; \mathbf{Z}\right)$. Then $\phi$ is written as $\phi=\prod \chi_{(f, g)}$ where the product ranges over all $f, g \in \mathbf{Z}_{n}\left[T, T^{-1}\right] /(h(T))$ such that $f(1)=i, g(1)=j$ for $i, j \in \mathbf{Z}_{n}$, where $n \mid h(1)$. We use this cocycle in the following theorem.

8.10. Theorem. For any positive integers $n, m>1$, there exists a cocycle in $C^{2}\left(\mathbf{Z}_{n}\left[T, T^{-1}\right] /\left(T^{2 m}-1\right) ; \mathbf{Z}\right)$ and a link $L$ whose cocycle invariant is non-trivial.

In particular, $H^{2}\left(\mathbf{Z}_{n}\left[T, T^{-1}\right] /\left(T^{2 m}-1\right) ; \mathbf{Z}\right) \neq 0$ for any $n, m>1$.

Proof. Let $L$ be the $(2 m n, 2)$-torus link, which is the closure of the braid $\sigma_{1}^{m n}$. If the elements $a, b$ are assigned as colors to the top of two strings of the braid, then after the $k$ th crossing, the colors assigned are $[a, b] B^{k}$ where $B$ is the Burau matrix $B=\left[\begin{array}{cc}0 & T \\ 1 & 1\end{array}\right]$. Each entry of the matrix $B^{2 m n}-I$ is divisible by the Alexander polynomial of $L$ (see [27] for example). The Alexander polynomial of $L$ is $\Delta=$ $T^{2 m n-1}-T^{2 m n-2}+\ldots-1$ (see for example [36]). With the relation $T^{2 m}=1$ and with the coefficients in $\mathbf{Z}_{n}, \Delta=0$. Hence $B^{2 m n}=I$ in $\mathbf{Z}_{n}\left[T, T^{-1}\right] /\left(T^{2 m}-1\right)$, and any pair $(a, b)$ gives a coloring of $L$. In particular, the pair $(0,1)$ defines a coloring and gives the term $T^{m n}$ with the cocycle $\phi_{0,1}$ defined in Remark 8.9.

8.11. Example. Let $L_{2 n}$ be the $(2 n, 2)$-torus link. We use the Alexander quandle $\mathbf{Z}_{3}\left[T, T^{-1}\right] /\left(T^{2}-1\right)$. Let $\phi=\phi_{0,1} \phi_{0,2}^{2} \phi_{1,2}^{3}$ where $\phi_{i, j}$ are defined in Remark 8.9] By listing the colorings, one computes that the cocycle invariant of $L_{2 n}$ is $27+$ $18\left(t^{3 n}+t^{6 n}+t^{9 n}\right)$ if $n=3 m$, and $9+6\left(t^{3 n}+t^{6 n}+t^{9 n}\right)$ otherwise. 
If we use the quandle $S_{4}=\mathbf{Z}_{2}\left[T, T^{-1}\right] /\left(T^{2}+T+1\right)$, then the trefoil and the figure 8 knot have non-trivial invariants using the 2-cocycle

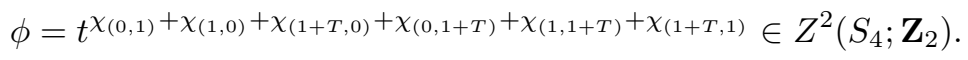

8.12. Theorem. The state-sum invariant for the trefoil and the figure 8 knot with the 2-cocycle $\phi$ defined above is

$$
4+12 t
$$

Proof. One can easily show that each knot can be colored in 16 ways using this quandle. The rest of the proof is a direct calculation.

Many other knots also can be seen to have the polynomial $4+12 t$ and its multiples as their invariants using this cocycle. It has also been computed that many knots in the knot table have non-trivial values with a different quandle than $S_{4}$.

\section{TRIPLE LINKING OF SURFACES AND COCYCLE INVARIANTS}

The linking number of a 2-component classical link $L=K_{1} \cup K_{2}$ can be defined by counting the crossing number with signs $( \pm 1)$ where the component $K_{1}$ crosses over $K_{2}$ ([38], see also the preceding section). This definition is generalized as follows to linked surfaces. Throughout this section, linked surfaces refer to oriented, multi-component, smoothly (or PL locally flatly) embedded surfaces in 4-space.

Recall from Definition 5.3 that the sign of a triple point is determined by comparing the ordered triple of vectors normal to the top, middle, and bottom sheets to the right-handed orientation of 3-space. Let $F=K_{1} \cup \cdots \cup K_{n}$ be a linked surface, where $K_{i}, i=1, \ldots, n$, are components.

9.1. Definition. Let $T_{ \pm}(i, j, k)$ denote the number of positive and negative, respectively, triple points such that the top, middle, and bottom sheets are from components $K_{i}, K_{j}$, and $K_{k}$ respectively. Such a triple point is called of type $(i, j, k)$. Then define $T(i, j, k)=T_{+}(i, j, k)-T_{-}(i, j, k)$.

9.2. Lemma. The numbers $T(i, j, k)$ are invariants of isotopy classes of $F$ if $i \neq j$ and $j \neq k$.

Proof. Consider the Roseman moves, depicted in Figure 7 that are analogues of the Reidemeister moves. The invariance of $T(i, j, k)$ is proved by checking that they remain unchanged under these moves.

More specifically, there are three moves involving triple points: (1) cancelation/creation of a pair of triple points (depicted in Figure 7 right top), (2) a branch point passing through a sheet (left bottom), and (3) the tetrahedral move, a move involving four planes (right bottom). In move (1), a pair of positive and negative triple points are involved, so that the number $T(i, j, k)$ remains unchanged. In move (2), the triple point involved is of type $(i, i, j)$ or $(i, j, j)$ because the branch point connects two sheets in the triple point, and these are the cases excluded in the theorem. The types of the various triple points remain the same on either side of move (3).

Thus these numbers are invariants of linked surfaces, which we call triple point linking invariants, or simply triple point invariants.

Although we provided a diagrammatic definition and proof, this invariant has been known in different contexts; see [44, 45, 32] for example. 
9.3. Theorem. For a linked oriented surface $L=K_{1} \cup \cdots \cup K_{n}$ and for any distinct $i, j \in\{1, \ldots, n\}$, we have $T(i, j, i)=0$.

Proof. Consider the double curves $D(i, j)$ where the over-sheet is $K_{i}$ and the undersheet is $K_{j}$. Then $D(i, j)$ is a set of immersed closed curves. The double curve is oriented in such a way that the ordered vectors of the normals $\vec{n}_{i}$ of $K_{i}$ and $\vec{n}_{j}$ of $K_{j}$ together with the direction $\vec{v}$ of $D(i, j)$ match the orientation of $\mathbf{R}^{3}$. Push each component of $D(i, j)$ off of $F$, to obtain a set of closed oriented curves $\gamma$ where the orientation is parallel to that of $D(i, j)$. Then the intersection number, $\gamma \cap K_{i}$, counted with sign is zero for a homological reason. Such intersections occur near triple points of type $(i, i, j)$ and $(i, j, i)$. Near each triple point of type $(i, i, j)$ a pair of intersections occurs, and they occur in cancelling signed pairs. However, near each triple point of type $(i, j, i)$ a single intersection occurs, and its sign matches the sign of the triple point. Therefore, $T(i, j, i)=0$.

9.4. Theorem. For a linked oriented surface $L=K_{1} \cup \cdots \cup K_{n}$ and for any triple $(i, j, k)$ with $i, j, k \in\{1, \ldots, n\}$ where $i, j$, and $k$ are distinct, it holds that

$$
T(i, j, k)-T(i, k, j)+T(k, i, j)=0 .
$$

Proof. The same argument as in the proof of Theorem 9.3, applied to $D(i, j)$ and $\gamma \cap K_{k}$ gives the equality. Note that the middle term receives a negative sign because of the sign conventions of intersection and that of triple points are opposite at these triple points.

The above conditions are equivalent to

9.5. Corollary. For any three component linked surface, there exist integers a and $b$ such that

$$
\begin{array}{lcccc}
T(1,2,3) & = & a & = & -T(3,2,1), \\
T(3,1,2) & = & b & = & -T(2,1,3), \\
T(2,3,1) & = & -(a+b) & = & -T(1,3,2) .
\end{array}
$$

9.6. Theorem. For any integers $a$ and $b$, there exists a linked surface $F=K_{1} \cup K_{2} \cup$ $K_{3}$ such that $T(i, j, k)(\{i, j, k\}=\{1,2,3\})$ satisfy the conditions in Corollary 9.5.

Proof. Consider the linked surface $F=K_{1} \cup K_{2} \cup K_{3}$ depicted in Figure12. Here $K_{1}$ is a sphere and $K_{2}$ and $K_{3}$ are tori. Two tori $K_{2}$ and $K_{3}$ intersect along two parallel double curves, such that $K_{2}$ is the over-sheet along one of them, and $K_{3}$ is over-sheet along the other. In other words, $K_{2} \cup K_{3}$ is a spun Hopf link. There are two intersections between $K_{1}$ and $K_{2} \cup K_{3}$. In the figure, at the bottom intersection $*$ is marked on the sphere, indicating that $K_{1}$ is the top sheet over all sheets of $K_{2} \cup K_{3}$, and in the top intersection, * is placed on $K_{2}$ to indicate that all sheets of $K_{2} \cup K_{3}$ are over $K_{1}$ (in other words $K_{1}$ is the bottom). Then the triple point invariants are computed as $T(1,2,3)=1, T(1,3,2)=1, T(2,3,1)=-1$, and $T(3,2,1)=-1$. This is the case where $a=1$ and $b=0$. An example of a linked surface with $a=0$ and $b=1$ is obtained by switching the components, and the cases $a=-1, b=0$ and $a=0, b=-1$ are obtained by changing orientations. The general case is obtained by taking the appropriate connected sum of copies of these examples. 


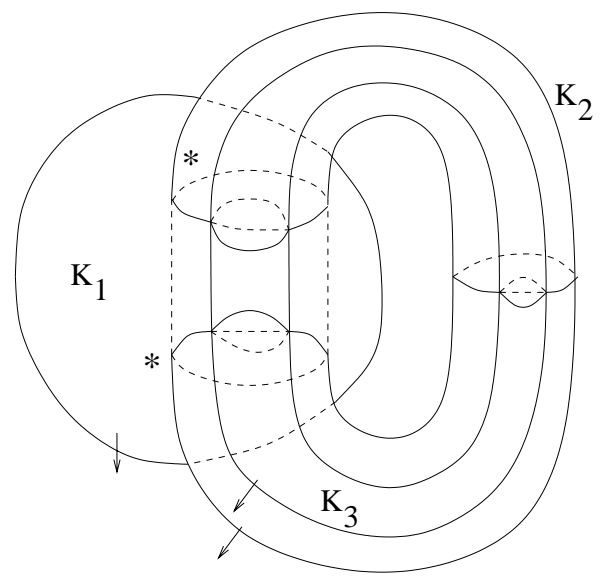

Figure 12. A surface link with non-trivial triple linking

9.7. Theorem. Let $X=\{x, y, z\}$ be the trivial quandle of three elements and $\theta \in Z^{3}(X ; \mathbf{Z})$ the cocycle $\chi_{(x, y, z)}$ which is the characteristic function

$$
\chi_{(x, y, z)}(p, q, r)= \begin{cases}t & \text { if }(p, q, r)=(x, y, z), \\ 1 & \text { otherwise. }\end{cases}
$$

For a linked surface of three connected components with triple point invariants as given in Corollary [9.5, the state-sum invariant is

$$
t^{a}+t^{-a}+t^{b}+t^{-b}+t^{a+b}+t^{-a-b}+21 .
$$

Proof. There are 27 ways of coloring the link: For a given connected component, each region of that component has the same color as all the other regions of that component.

If a coloring uses fewer than three colors, then it contributes the value 1 as a term in the state-sum. On the other hand, for a given coloring $\mathcal{C}$, say $\mathcal{C}\left(K_{i}\right)=z$, $\mathcal{C}\left(K_{j}\right)=y, \mathcal{C}\left(K_{k}\right)=x$, the Boltzmann weight of a triple point is $t$ if and only if the triple point is positive and of type $(i, j, k)$. The weight is $t^{-1}$ if and only if the triple point is negative of the same type. The weight is 1 otherwise. So this coloring contributes a term $t^{T(i, j, k)}$ to the state-sum.

9.8. Remark. The same argument as above, together with Theorem 9.3 gives that when the two-element trivial quandle, $T_{2}$, is used, the state-sum invariant associated with any 3 -cocycle is trivial.

\section{SURFACE BRAIDS AND QUANDLES}

In this section we give a method to obtain a presentation of the quandle of a surface braid described by a chart, which is used in order to calculate the state-sum invariants of surfaces in 4-space.

Let $D^{2}$ and $D$ be 2-disks and $X_{m}$ a fixed set of $m$ interior points of $D^{2}$. By $p r_{1}: D^{2} \times D \rightarrow D^{2}$ and $p r_{2}: D^{2} \times D \rightarrow D$, we mean the projections to the first factor and to the second factor, respectively.

10.1. Definition. A surface braid $([23,43])$ of degree $m$ is a compact, oriented surface $S$ properly embedded in $D^{2} \times D$ such that the restriction of $p r_{2}$ to $S$ is 
a degree- $m$ simple branched covering map and $\partial S=X_{m} \times \partial D \subset D^{2} \times \partial D$. A degree- $m$ branched covering map $f: S \rightarrow D$ is simple if $\left|f^{-1}(y)\right|=m$ or $m-1$ for $y \in D$. In this case, the branch points are simple $\left(z \mapsto z^{2}\right)$.

A surface braid $S$ of degree $m$ is extended to a closed surface $\widehat{S}$ in $D^{2} \times S^{2}$ such that $\widehat{S} \cap\left(D^{2} \times D\right)=S$ and $\widehat{S} \cap\left(D^{2} \times \bar{D}\right)=X_{m} \times \bar{D}$, where $S^{2}$ is the 2-sphere obtained from $D^{2}$ by attaching a 2-disk $\bar{D}$ along the boundary. By identifying $D^{2} \times S^{2}$ with the tubular neighborhood of a standard 2-sphere in $\mathbf{R}^{4}$, we assume that $\widehat{S}$ is a closed oriented surface embedded in $\mathbf{R}^{4}$. We call it the closure of $S$ in $\mathbf{R}^{4}$. It is proved in [24] that every closed oriented surface embedded in $\mathbf{R}^{4}$ is ambient isotopic to the closure of a surface braid.

Two surface braids $S$ and $S^{\prime}$ in $D^{2} \times D$ are said to be equivalent if there is an isotopy $\left\{h_{t}\right\}$ of $D^{2} \times D$ such that

(1) $h_{0}=\mathrm{id}, h_{1}(S)=S^{\prime}$,

(2) for each $t \in[0,1], h_{t}$ is fiber-preserving; that is, there is a homeomorphism $\underline{h}_{t}: D \rightarrow D$ with $\underline{h}_{t} \circ p r_{2}=p r_{2} \circ h_{t}$, and

(3) for each $t \in[0,1],\left.h_{t}\right|_{D^{2} \times \partial D}=\mathrm{id}$.

Let $C_{m}$ be the configuration space of unordered $m$ interior points of $D^{2}$. We identify the fundamental group $\pi_{1}\left(C_{m}, X_{m}\right)$ of $C_{m}$ with base point $X_{m}$ with the braid group $B_{m}$ on $m$ strings. Let $S$ denote a surface braid and $\Sigma(S) \subset D$ the branch point set of the branched covering map $S \rightarrow D$. For a path $a:[0,1] \rightarrow$ $D \backslash \Sigma(S)$, we define a path

$$
\rho_{S}(a):[0,1] \rightarrow C_{m}
$$

by

$$
\rho_{S}(a)(t)=\operatorname{pr}_{1}\left(S \cap\left(D^{2} \times\{a(t)\}\right)\right) .
$$

If $\operatorname{pr}_{1}\left(S \cap\left(D^{2} \times\{a(0)\}\right)\right)=\operatorname{pr}_{1}\left(S \cap\left(D^{2} \times\{a(1)\}\right)\right)=X_{m}$, then the path $\rho_{S}(a)$ represents an element of $\pi_{1}\left(C_{m}, X_{m}\right)=B_{m}$. Take a point $y_{0}$ in $\partial D$. The braid monodromy of $S$ is the homomorphism

$$
\rho_{S}: \pi_{1}\left(D \backslash \Sigma(S), y_{0}\right) \rightarrow B_{m}
$$

such that $\rho_{S}([a])=\left[\rho_{S}(a)\right]$ for any loop $a$ in $D \backslash \Sigma(S)$ with base point $y_{0}$.

Let $\Sigma(S)=\left\{y_{1}, \ldots, y_{n}\right\}$. Take a regular neighborhood $N(\Sigma(S))=N\left(y_{1}\right) \cup \cdots \cup$ $N\left(y_{n}\right)$ in $D$. A Hurwitz arc system $\mathcal{A}=\left(\alpha_{1}, \ldots, \alpha_{n}\right)$ for $\Sigma(S)$ is an $n$-tuple of simple arcs in $E(\Sigma(S))=\mathrm{Cl}(D \backslash N(\Sigma(S))$ ) (where $\mathrm{Cl}$ denotes the closure) such that each $\alpha_{i}$ starts from a point of $\partial N\left(y_{i}\right)$ and ends at $y_{0}$, and $\alpha_{i} \cap \alpha_{j}=\left\{y_{0}\right\}$ for $i \neq j$, and $\alpha_{1}, \ldots, \alpha_{n}$ appear in this order around $y_{0}$.

Let $\eta_{i}(i=1, \ldots, n)$ be the loop $\alpha_{i}^{-1} \cdot \partial N\left(y_{i}\right) \cdot \alpha_{i}$ in $D \backslash \Sigma(S)$ with base point $y_{0}$ which goes along $\alpha_{i}$, turns along $\partial N\left(y_{i}\right)$ in the positive direction, and returns along $\alpha_{i}$.

10.2. Definition. The braid system of $S$ associated with $\mathcal{A}$ is an $n$-tuple of $m$-braids

$$
\left(\rho_{S}\left(\left[\eta_{1}\right]\right), \rho_{S}\left(\left[\eta_{2}\right]\right), \ldots, \rho_{S}\left(\left[\eta_{n}\right]\right)\right) .
$$

Each element of a braid system is a conjugate of a standard generator $\sigma_{i}$ of $B_{m}$ or its inverse. The braid system of a surface braid of degree $m$ is written as

$$
\left(w_{1}^{-1} s_{1}^{\epsilon_{1}} w_{1}, w_{2}^{-1} s_{2}^{\epsilon_{2}} w_{2}, \ldots, w_{n}^{-1} s_{n}^{\epsilon_{n}} w_{n}\right),
$$

where $n$ is the number of branch points, $w_{1}, \ldots, w_{n}$ are $m$-braids, $s_{1}, \ldots, s_{n} \in$ $\left\{\sigma_{1}, \ldots, \sigma_{m-1}\right\}$ and $\epsilon_{1}, \ldots, \epsilon_{n} \in\{+1,-1\}$. 
10.3. Definition. An $m$-chart [23] is an oriented, labelled graph $\Gamma$ in $D$, which may be empty or have closed edges without vertices (which are called hoops), satisfying the following conditions:

(1) Every vertex has degree one, four or six.

(2) The labels of edges are in $\{1,2, \ldots, m-1\}$.

(3) For each degree-six vertex, three consective edges are oriented inward and the other three are outward, and these six edges are labelled $i$ and $i+1$ alternately for some $i$.

(4) For each degree-four vertex, diagonal edges have the same label and are oriented coherently, and the labels $i$ and $j$ of the diagonals satisfy $|i-j|>1$.

We call a degree 1 (resp. degree 6) vertex a black (resp. white) vertex. A degree 4 vertex is called a crossing point of the chart.

We say that a path $\alpha:[0,1] \rightarrow D$ is in general position with respect to $\Gamma$ if it avoids the vertices of $\Gamma$ and every intersection of $\alpha$ and $\Gamma$ is a transverse double point. If $p$ is an intersection of $\alpha$ and an edge of $\Gamma$ labelled $i$ and if the edge is oriented from right to left (resp. from left to right), then assign the intersection $p$ a letter $\sigma_{i}$ (resp. $\left.\sigma_{i}^{-1}\right)$. Read the letters assigned to the intersections of $\alpha$ and $\Gamma$ along $\alpha$ and we have a word

$$
\sigma_{i_{1}}^{\epsilon_{1}} \sigma_{i_{2}}^{\epsilon_{2}} \ldots \sigma_{i_{s}}^{\epsilon_{s}}
$$

in the braid generator. We call this the intersection braid word of $\alpha$ with respect to $\Gamma$, and denote it by $w_{\Gamma}(\alpha)$.

For an $m$-chart $\Gamma$, a surface braid described by $\Gamma$ means a surface braid $S$ of degree $m$ satisfying the following conditions:

(1) For a regular neighborhood $N(\Gamma)$ of $\Gamma$ in $D$ and for any $y \in \operatorname{Cl}(D \backslash N(\Gamma))$, the projection $p r_{1}$ satisfies the condition $\operatorname{pr}_{1}\left(S \cap\left(D^{2} \times\{y\}\right)\right)=X_{m}$, where $X_{m}$ denotes the $m$ fixed interior points of $D^{2}$.

(2) The branch point set of $S$ corresponds to the set of the black vertices of $\Gamma$.

(3) For a path $\alpha:[0,1] \rightarrow D$ which is in general position with respect to $\Gamma$ and $\alpha(0), \alpha(1)$ are in $\operatorname{Cl}(D \backslash N(\Gamma))$, the $m$-braid determined by $\rho_{S}(\alpha)$ is the $m$-braid presented by the intersection braid word $w_{\Gamma}(a)$.

10.4. Proposition. (1) For any $m$-chart $\Gamma$, there is a unique (up to equivalence) surface braid described by $\Gamma$.

(2) For any surface braid $S$ of degree $m$, there is an $m$-chart $\Gamma$ such that $S$ is equivalent to a surface braid described by $\Gamma$.

Let $S$ be a surface braid described by $\Gamma$. Identify $D^{2}$ with $I_{1} \times I_{2}$ and $D$ with $I_{3} \times I_{4}$, where $I_{i}(i=1, \ldots, 4)$ are intervals. For each $t \in I_{4}$, put $b_{t}=$ $S \cap\left(D^{2} \times I_{3} \times\{t\}\right)$. Then $\left\{b_{t} \mid t \in I_{4}\right\}$ is a continuous sequence of $m$-braids with a finite number of exceptions that are singular $m$-braids. Modifying $\Gamma$ by an ambient isotopy of $D$, we may assume that every white vertex $W$ looks like one of the Figure 13 with respect to the bi-parametrization $D \cong I_{3} \times I_{4}$. Then the sequence $\left\{b_{t}\right\}$ looks like the motion pictures in Figure 14 around the white vertex.

We assume that each $b_{t}$ is illustrated as a diagram with respect to the projection $I_{1} \times I_{2} \times I_{3} \rightarrow I_{2} \times I_{3}$. Then under the projection $I_{1} \times I_{2} \times I_{3} \times I_{4} \rightarrow I_{2} \times I_{3} \times I_{4}$, the image of $S$ has a triple point corresponding to a white vertex. We define the sign $\epsilon(W)$ of a white vertex, $W$, by +1 (resp. -1) if it is as (A) (resp. as (B)) in Figure 13 so that the corresponding triple point has sign +1 (resp. -1) in the broken surface diagram of $S$. (In general, the singularity set of the image of $S$ by 


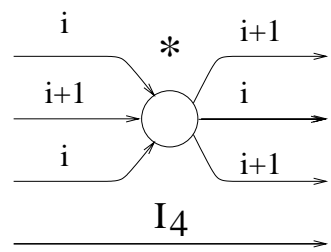

A

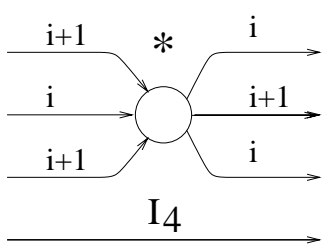

B

FIgURE 13. The distinguished region of a white vertex

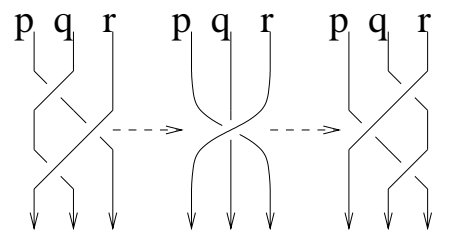

A

i i +1 i+2 i i+1 i+2 i i+1 i+2

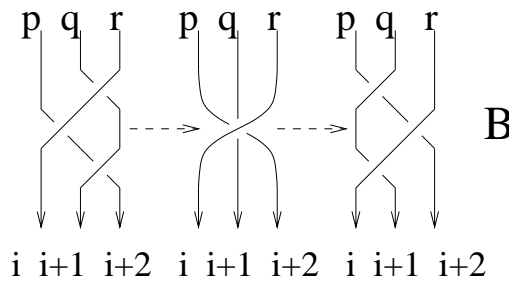

Figure 14. Quandle labels near a white vertex

the projection $I_{1} \times I_{2} \times I_{3} \times I_{4} \rightarrow I_{2} \times I_{3} \times I_{4}$ is identified naturally with the chart $\Gamma$ in the sense of 7 , 25]. The white vertices are in one-to-one correspondence to the triple points and the black vertices are in one-to-one correspondence to the branch points. Figure 15] shows the relationship schematically; see [7, 25] for details.)

When a surface braid is described by a chart, the braid system is easily obtained as follows:

10.5. Lemma. Let $S$ be a surface braid described by a chart $\Gamma$, and let $\mathcal{A}=$ $\left(\alpha_{1}, \ldots, \alpha_{n}\right)$ be a Hurwitz arc system for $\Sigma(S)$ such that each $\alpha_{i}$ is in general position with respect to $\Gamma$. The braid system of $S$ associated with $\mathcal{A}$ is given by

$$
\left(w_{\Gamma}\left(\eta_{1}\right), w_{\Gamma}\left(\eta_{2}\right), \ldots, w_{\Gamma}\left(\eta_{n}\right)\right)
$$

where $\eta_{1}, \ldots, \eta_{n}$ are loops in $D \backslash \Sigma(S)$ associated with $\mathcal{A}$ as before.

Proof. By definition of $S, \rho_{S}\left(\left[\eta_{i}\right]\right)=w_{\Gamma}\left(\eta_{i}\right)$ for $i=1, \ldots, n$.

10.6. Example. Let $\Gamma$ be the 4-chart as in Figure 16 and $S$ a surface braid of degree 4 described by $\Gamma$. It is known that this chart represents the 2-twist spun trefoil knot [23]. Recall that every black vertex stands for a branch point of $S \rightarrow D$. Let $\mathcal{A}=\left(\alpha_{1}, \ldots, \alpha_{n}\right)$ be a Hurwitz arc system for $\Sigma(S)$ illustrated in the figure, where 


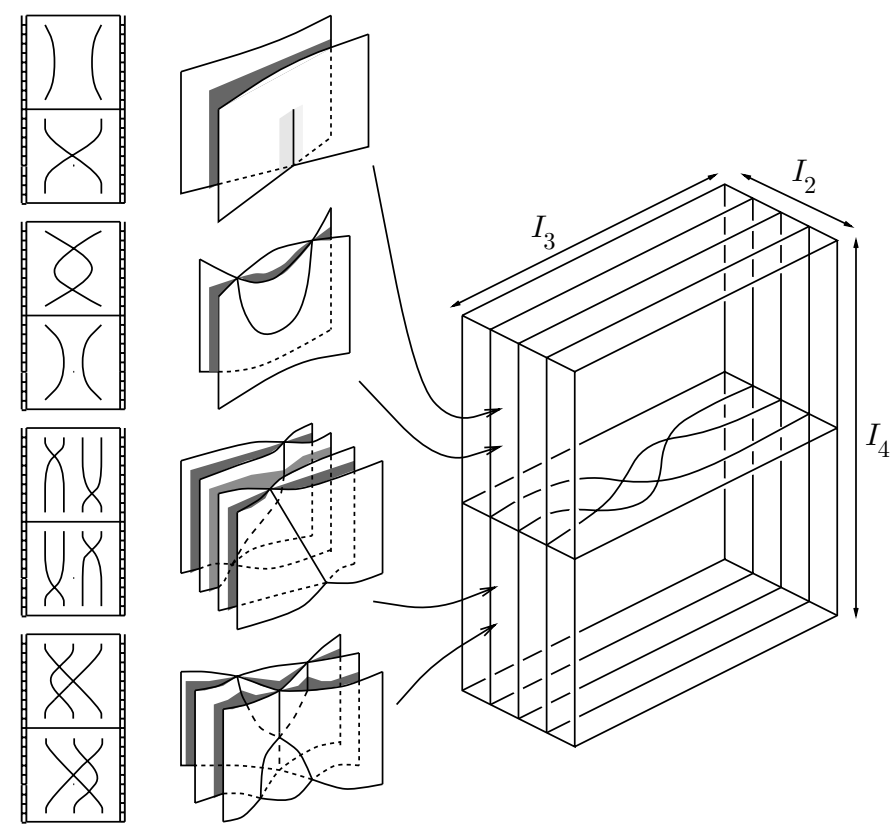

Figure 15. Projections and charts

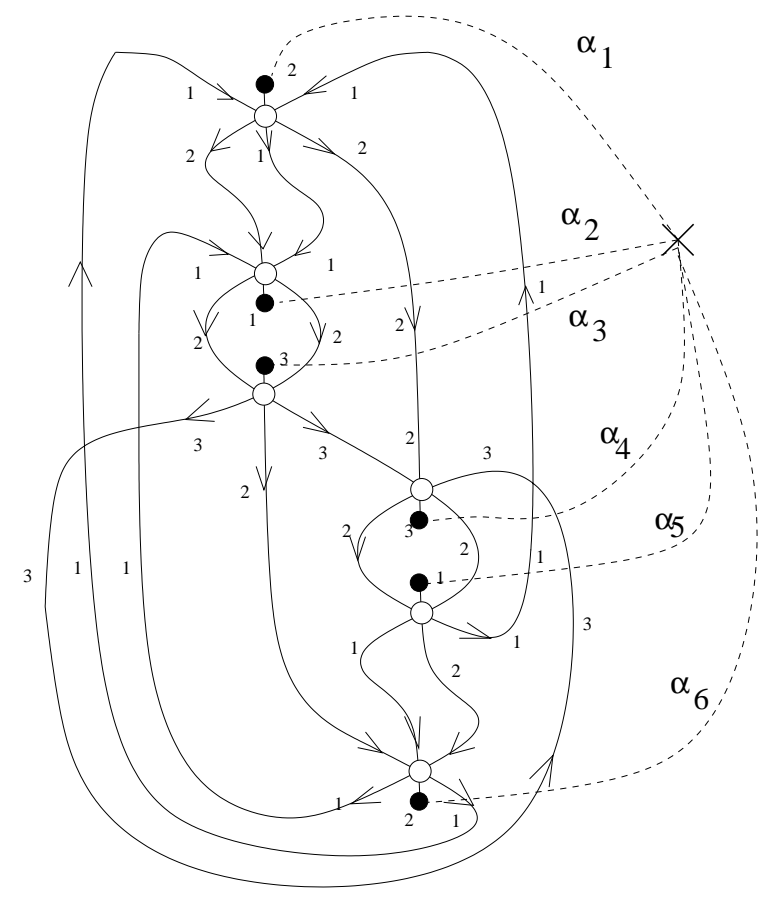

FiguRE 16. A Hurwitz system of a chart 

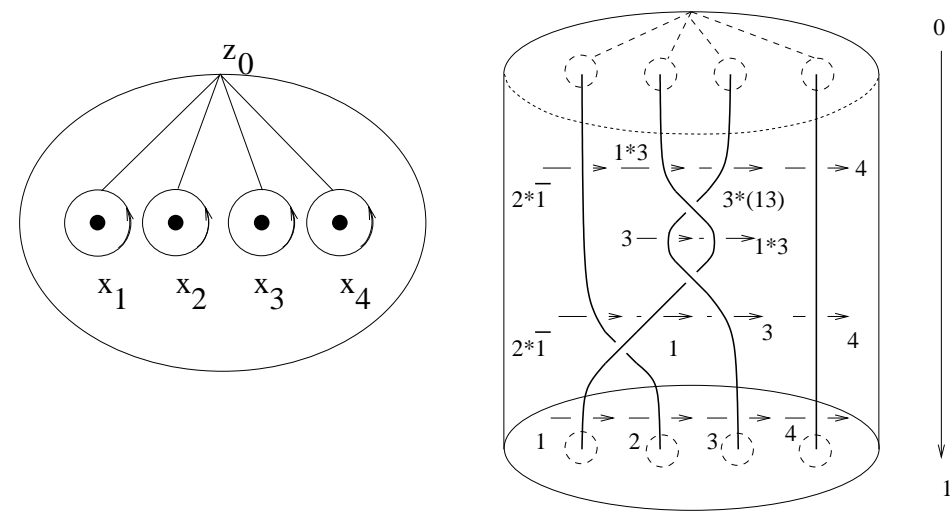

Figure 17. Generators of the free quandle

$\alpha_{1}, \ldots, \alpha_{6}$ are drawn as dotted arcs. The braid system $\left(w_{1}^{-1} \sigma_{k_{1}}^{\epsilon_{1}} w_{1}, w_{2}^{-1} \sigma_{k_{2}}^{\epsilon_{2}} w_{2}, \ldots\right.$, $\left.w_{6}^{-1} \sigma_{k_{6}}^{\epsilon_{6}} w_{6}\right)$ of $S$ is given by

$$
\begin{array}{ll}
w_{1}=1, & \sigma_{k_{1}}^{\epsilon_{1}}=\sigma_{2}^{-1}, \\
w_{2}=\sigma_{2}^{-2} \sigma_{1}, & \sigma_{k_{2}}^{\epsilon_{2}}=\sigma_{1}, \\
w_{3}=\sigma_{2}^{-2} \sigma_{1}, & \sigma_{k_{3}}^{\epsilon_{3}}=\sigma_{3}^{-1}, \\
w_{4}=\sigma_{2}^{-1} \sigma_{1} \sigma_{3}, & \sigma_{k_{4}}^{\epsilon_{4}}=\sigma_{3}, \\
w_{5}=\sigma_{2}^{-1} \sigma_{1} \sigma_{3}, & \sigma_{k_{5}}^{\epsilon_{5}}=\sigma_{1}^{-1}, \\
w_{6}=\sigma_{1}^{-1} \sigma_{3}, & \sigma_{k_{6}}^{\epsilon_{6}}=\sigma_{2} .
\end{array}
$$

10.7. Definition ([12, 20]). Let $M$ be an oriented $(n+2)$-manifold, and $L$ an oriented $n$-submanifold of $M$ with a tubular neighborhood $N(L)$ in $M$. Take a point $z \in E(L)=\mathrm{Cl}(M \backslash N(L))$. Consider the set of paths $\alpha:[0,1] \rightarrow E(L)$ such that there is a meridian disk, say $\Delta_{\alpha}$, of $L$ with $\alpha(0) \in \partial \Delta_{\alpha}$ and $\alpha(1)=z$. Let $Q(M, L, z)$ be the set of homotopy classes of paths $\alpha$. Define a binary operation $*$ on $Q(M, L, z)$ by

$$
[\alpha] *[\beta]=\left[\alpha \cdot \beta^{-1} \cdot \partial \Delta_{\beta} \cdot \beta\right]
$$

where $\Delta_{\beta}$ is an (oriented) meridian disk with $\beta(0) \in \partial \Delta_{\beta}$. Then $Q(M, L, z)$ with $*$ is a quandle, which is called the quandle of $(M, L)$, or the quandle of $L$, with base point $z$, and denoted by $Q(M, L, z)$ (or $Q(M, L), Q(L)$, etc.).

10.8. Example. Let $b$ be an $m$-braid, and let $f_{b}:\left(D^{2}, X_{m}\right) \rightarrow\left(D^{2}, X_{m}\right)$ be an Artin homeomorphism associated with $b$. We denote by $Q(b)$ the quandle isomorphism

$$
\left(f_{b}\right)_{*}: Q\left(D^{2}, X_{m}, z_{0}\right) \rightarrow Q\left(D^{2}, X_{m}, z_{0}\right)
$$

induced from the Artin homeomorphism $f_{b}$. We usually identify $Q\left(D^{2}, X_{m}, z_{0}\right)$ with the free quandle $F_{Q}\left\langle x_{1}, \ldots, x_{m}\right\rangle$ generated by $x_{1}, \ldots, x_{m}$ as in Figure 17 and regard $Q(b)$ as a quandle automorphism of the free quandle $F_{Q}\left\langle x_{1}, \ldots, x_{m}\right\rangle$.

The quandle automorphism $Q(b)$ is interpreted as follows: Let $\alpha$ be the path in $D^{2} \times[0,1]$ defined by $\alpha(t)=\left(z_{0}, t\right)$. We have a quandle isomorphism

$$
\alpha_{*}: Q\left(D^{2} \times[0,1], b, z_{0} \times\{0\}\right) \rightarrow Q\left(D^{2} \times[0,1], b, z_{0} \times\{1\}\right)
$$


such that $\alpha_{*}([\beta])=[\beta \cdot \alpha]$. Since the inclusion-induced quandle homomorphisms

$$
\left(i_{j}\right)_{*}: Q\left(D^{2} \times\{j\}, X_{m} \times\{j\}, z_{0} \times\{j\}\right) \rightarrow Q\left(D^{2} \times[0,1], b, z_{0} \times\{j\}\right) \quad(j=0,1)
$$

are isomorphisms, we have an isomorphism

$$
\left(i_{1}\right)_{*}^{-1} \circ \alpha_{*} \circ\left(i_{0}\right)_{*}: Q\left(D^{2} \times\{0\}, X_{m} \times\{0\}, z_{0} \times\{0\}\right) \rightarrow Q\left(D^{2} \times\{1\}, X_{m} \times\{1\}, z_{0} \times\{1\}\right) .
$$

Identifying $Q\left(D^{2} \times\{j\}, X_{m} \times\{j\}, z_{0} \times\{j\}\right)(j=0,1)$ with $Q\left(D^{2}, X_{m}, z_{0}\right)$ via the projection $D^{2} \times[0,1] \rightarrow D^{2}$, we have an automorphism of $Q\left(D^{2}, X_{m}, z_{0}\right)$. This is $Q(b)$.

For example, if $b=\sigma_{2}^{-2} \sigma_{1} \in B_{4}$, then the quandle $Q\left(D^{2}, X_{4}, z_{0}\right)$ is freely generated by $x_{1}, \ldots, x_{4}$ as illustrated in Figure 17] and the quandle isomorphism $Q(b)$ maps the generators as follows:

$$
\begin{aligned}
& Q(b)\left(x_{1}\right)=x_{2} * x_{1}^{-1} \\
& Q(b)\left(x_{2}\right)=x_{1} * x_{3} \\
& Q(b)\left(x_{3}\right)=x_{3} *\left(x_{1} x_{3}\right) \\
& Q(b)\left(x_{4}\right)=x_{4}
\end{aligned}
$$

In the above table and in the sequel, we are mimicking the notation in 12. So, $a *(b c)$ is defined to be $(a * b) * c$; the element $a *\left(b^{-1}\right)$ is the unique element $c$ such that $a=c * b$; and generally a product $a * w$ where $w$ is a word on the free group generated by the quandle can be interpreted inductively. For example, $x_{1} *\left(x_{3} x_{4} x_{3}^{-1}\right)=\left(\left(x_{1} * x_{3}\right) * x_{4}\right) * x_{3}^{-1}$. See also Figure 17

10.9. Lemma. Let $\left(b_{1}, \ldots, b_{n}\right)$ be a braid system of the surface braid $S$, then the quandle $Q(S)$ has a presentation whose generators are $x_{1}, \ldots, x_{m}$ and the relations are

$$
Q\left(w_{i}\right)\left(x_{k_{i}}\right)=Q\left(w_{i}\right)\left(x_{k_{i}+1}\right) \quad(i=1, \ldots, n),
$$

where $b_{i}=w_{i}^{-1} \sigma_{k_{i}}^{\epsilon_{i}} w_{i}$.

Proof. In [43] and 24] it is shown that the fundamental group $\pi_{1}\left(D^{2} \times D \backslash S, z_{0} \times y_{0}\right)$ is generated by $m$ positive meridional elements $x_{1}, \ldots, x_{m}$ with defining relations

$$
\left(f_{w_{i}}\right)_{*}\left(x_{k_{i}}\right)=\left(f_{w_{i}}\right)_{*}\left(x_{k_{i}+1}\right) \quad(i=1, \ldots, n),
$$

where $\left(f_{w_{i}}\right)_{*}: \pi_{1}\left(D^{2} \backslash X_{m}, z_{0}\right) \rightarrow \pi_{1}\left(D^{2} \backslash X_{m}, z_{0}\right)$ is the automorphism induced from the Artin homeomorphism $f_{w_{i}}:\left(D^{2}, X_{m}, z_{0}\right) \rightarrow \pi_{1}\left(D^{2}, X_{m}, z_{0}\right)$ associated with the braid $w_{i}$. In [12, 20], presentations of quandles of codimension 2 embeddings in Euclidean spaces were given that are similar to Wirtinger presentations of fundamental groups. Thus a similar argument as above gives the presentation of $Q(S)$

10.10. Example. Let $S$ be the surface braid of degree 4 described by a 4 -chart $\Gamma$ in Figure 16, For a Hurwitz arc system $\mathcal{A}=\left(\alpha_{1}, \ldots, \alpha_{6}\right)$ as in Figure 16 the braid system $\left(w_{1}^{-1} \sigma_{k_{1}}^{\epsilon_{1}} w_{1}, \ldots, w_{6}^{-1} \sigma_{k_{6}}^{\epsilon_{6}} w_{6}\right)$ of $S$ is given as in Example 10.6. The quandle automorphisms $Q(1), Q\left(\sigma_{2}^{-2} \sigma_{1}\right), Q\left(\sigma_{2}^{-1} \sigma_{1} \sigma_{3}\right)$, and $Q\left(\sigma_{1}^{-1} \sigma_{3}\right)$ of $F_{Q}\left\langle x_{1}, \ldots, x_{m}\right\rangle$ 
map the generators as follows:

$$
\begin{aligned}
Q(1) & : x_{1} \mapsto x_{1}, \quad x_{2} \mapsto x_{2}, \quad x_{3} \mapsto x_{3}, \quad x_{4} \mapsto x_{4}, \\
Q\left(\sigma_{2}^{-2} \sigma_{1}\right) & : \quad x_{1} \mapsto x_{2} * x_{1}^{-1}, \quad x_{2} \mapsto x_{1} * x_{3}, \quad x_{3} \mapsto x_{3} *\left(x_{1} x_{3}\right), \quad x_{4} \mapsto x_{4}, \\
Q\left(\sigma_{2}^{-1} \sigma_{1} \sigma_{3}\right) & : \quad x_{1} \mapsto x_{2} * x_{1}^{-1}, \quad x_{2} \mapsto x_{4} * x_{3}^{-1}, \quad x_{3} \mapsto x_{1} *\left(x_{3} x_{4} x_{3}^{-1}\right), \quad x_{4} \mapsto x_{3}, \\
Q\left(\sigma_{1}^{-1} \sigma_{3}\right) & : \quad x_{1} \mapsto x_{2}, \quad x_{2} \mapsto x_{1} * x_{2}, \quad x_{3} \mapsto x_{4} * x_{3}^{-1}, \quad x_{4} \mapsto x_{3} .
\end{aligned}
$$

Hence the defining relations $Q\left(w_{i}\right)\left(x_{k_{i}}\right)=Q\left(w_{i}\right)\left(x_{k_{i}+1}\right)(i=1, \ldots, 6)$ of $Q(S)$ are

$$
\begin{aligned}
x_{2} & =x_{3}, \\
x_{2} * x_{1}^{-1} & =x_{1} * x_{3}, \\
x_{3} *\left(x_{1} x_{3}\right) & =x_{4}, \\
x_{1} *\left(x_{3} x_{4} x_{3}^{-1}\right) & =x_{3}, \\
x_{2} * x_{1}^{-1} & =x_{4} * x_{3}^{-1}, \\
x_{1} * x_{2} & =x_{4} * x_{3}^{-1} .
\end{aligned}
$$

Thus the quandle $Q(S)$ is

$$
\begin{aligned}
\left\langle x_{1}, \ldots, x_{4}\right| & x_{2}=x_{1} *\left(x_{2} x_{1}\right), \\
& x_{2}=x_{2} *\left(x_{1}^{2}\right), \\
& x_{3}=x_{2}, \\
& \left.x_{4}=x_{1}\right\rangle \\
=\left\langle x_{1}, x_{2}\right| & x_{2}=x_{1} *\left(x_{2} x_{1}\right), \\
& \left.x_{2}=x_{2} *\left(x_{1}^{2}\right)\right\rangle .
\end{aligned}
$$

\section{CocyCle InVARIANTS AND BRAID CHARTS}

In this section we introduce a method to calculate the state-sum invariant of a surface braid described by a chart. The state-sum invariant of a surface braid coincides with the state-sum invariant of its closure in $\mathbf{R}^{4}$.

Let $S$ be a surface braid of degree $m$ described by an $m$-chart $\Gamma$. The region of $D \backslash \Gamma$ assigned the asterisk in Figure 13 is called the distinguished region for a white vertex $W$. Let $y$ be a point of this region. Since $S$ is a surface braid described by $\Gamma$, we may assume that $\operatorname{pr}_{1}\left(S \cap\left(D^{2} \times\{y\}\right)\right)=X_{m}$. Then $Q_{y}=$ $Q\left(D^{2} \times\{y\}, X_{m} \times\{y\}, z_{0} \times\{y\}\right)$ is identified with $Q\left(D^{2}, X_{m}, z_{0}\right)=F_{Q}\left\langle x_{1}, \ldots, x_{m}\right\rangle$ via the projection $p r_{1}$. Take a path $\beta:[0,1] \rightarrow D \backslash \Sigma(S)$ with $\beta(0)=y$ and $\beta(1)=y_{0}$. The $m$-braid $\rho_{S}(\beta)$ induces an isomorphism

$$
Q\left(\rho_{S}(\beta)\right): F_{Q}\left\langle x_{1}, \ldots, x_{m}\right\rangle=Q_{y} \rightarrow Q_{y_{0}}=F_{Q}\left\langle x_{1}, \ldots, x_{m}\right\rangle
$$

Recall that the generators $x_{1}, \ldots, x_{m}$ of the quandle $Q(S)=Q\left(D^{2} \times D, S, z_{0} \times y_{0}\right)$ in Lemma 10.9 are the images $i_{*}\left(x_{1}\right), \ldots, i_{*}\left(x_{m}\right)$ of $x_{1}, \ldots, x_{m} \in Q_{y_{0}}$ by the inclusioninduced homomorphism $i_{*}: Q_{y_{0}} \rightarrow Q(S)$.

Let $p, q, r$ be the elements of $Q(S)$ corresponding to the three sheets in the broken surface diagram of $S$ as in the motion pictures depicted in Figure 14. Then

$$
\begin{aligned}
p & =i_{*} \circ Q\left(\rho_{S}(\beta)\right)\left(x_{i}\right), \\
q & =i_{*} \circ Q\left(\rho_{S}(\beta)\right)\left(x_{i+1}\right), \\
r & =i_{*} \circ Q\left(\rho_{S}(\beta)\right)\left(x_{i+2}\right) .
\end{aligned}
$$


We call $(p, q, r)$ the quandle triple for the white vertex $W$ or for the triple point corresponding to $W$.

Let $\theta \in Z^{3}(X ; A)$ be a 3 -cocycle of a finite quandle $X$ with coefficient group $A$ and let $c: Q(S)=Q\left(D^{2} \times D, S, z_{0} \times y_{0}\right) \rightarrow X$ be a homomorphism (a coloring). We define the Boltzmann weight on a white vertex $W$ by

$$
\theta(c(p), c(q), c(r))^{\epsilon(W)},
$$

where $\epsilon(W)$ is the sign of $W$, and $(p, q, r)$ is the quandle triple for $W$, and put

$$
\Phi_{\theta}(\Gamma)=\sum_{c} \prod_{W} \theta(c(p), c(q), c(r))^{\epsilon(W)},
$$

where $W$ runs over all white vertex of the chart $\Gamma$ and $c$ runs over all possible coloring from $Q(S)$ to $X$.

11.1. Lemma. Let $S$ be a surface braid described by a chart $\Gamma$ and let $\widehat{S}$ be the closure of $S$ in $\mathbf{R}^{4}$. Then

$$
\Phi_{\theta}(\Gamma)=\Phi_{\theta}(\widehat{S})
$$

Proof. Recall the situation depicted in Figure 15. Consider a broken surface diagram of $S$ by the projection $I_{1} \times I_{2} \times I_{3} \times I_{4} \rightarrow I_{2} \times I_{3} \times I_{4}$. The broken surface diagram of $\widehat{S}$ is obtained from the diagram of $S$ by attaching $m$ disks outside of $I_{2} \times I_{3} \times I_{4}$ trivially. So there is a one-to-one correspondence between the colorings of them. Every white vertex corresponds to a triple point, and the Boltzmann weight of a white vertex is the same with that of the triple point. (In fact, we defined it to be so.) Hence $\Phi_{\theta}(\Gamma)=\Phi_{\theta}(\widehat{S})$.

11.2. Theorem. Let $F$ be the 2-twist spun trefoil and $\theta$ a quandle 3-cocycle of a finite quandle $X$ with coefficient group $A$. The state-sum invariant $\Phi_{\theta}(F)$ is

$$
\begin{aligned}
\sum_{y_{1}, y_{2}} \theta & \left(y_{1} * y_{2}, y_{1}, y_{2}\right) \theta\left(y_{1} * y_{2}, y_{2}, y_{1} * y_{2}\right) \theta\left(y_{2}, y_{1} * y_{2}, y_{1}\right) \\
& \times \theta\left(y_{1}, y_{1} * y_{2}, y_{2}\right)^{-1} \theta\left(y_{1} * y_{2}, y_{1}, y_{1} * y_{2}\right)^{-1} \theta\left(y_{1}, y_{2}, y_{1} * y_{2}\right)^{-1},
\end{aligned}
$$

where $y_{1}, y_{2}$ run over all elements of $X$ satisfying $y_{2}=y_{1} *\left(y_{2} y_{1}\right)$ and $y_{2}=y_{2} *\left(y_{1}^{2}\right)$.

Proof. Let $S$ be a surface braid of degree 4 described by the 4 -chart $\Gamma$ in Figure 16 (Figure 18). Let $\beta_{1}, \ldots, \beta_{6}$ be the paths from points in the distinguished regions of the white vertices as in Figure 18 Let $W_{i}$ be the white vertex near the end of $\beta_{i}$, for $i=1, \ldots, 6$. The 4 -braids $\rho_{S}\left(\beta_{1}\right), \ldots, \rho_{S}\left(\beta_{6}\right)$ are represented by the intersection braid words $w_{\Gamma}\left(\beta_{1}\right), \ldots, w_{\Gamma}\left(\beta_{6}\right)$, which are

$$
\sigma_{1}, \quad \sigma_{2}^{-1} \sigma_{1}, \quad \sigma_{2}^{-1} \sigma_{1}, \quad \sigma_{1} \sigma_{3}, \quad \sigma_{1} \sigma_{3}, \quad \text { and } \sigma_{3},
$$

respectively.

The quandle automorphisms

$$
Q\left(\sigma_{1}\right), \quad Q\left(\sigma_{2}^{-1} \sigma_{1}\right), \quad Q\left(\sigma_{1} \sigma_{3}\right) \text { and } Q\left(\sigma_{3}\right)
$$

of $F_{Q}\left\langle x_{1}, \ldots, x_{m}\right\rangle$ map the generators as follows:

$$
\begin{array}{rlll}
Q\left(\sigma_{1}\right) & : & x_{1} \mapsto x_{2} * x_{1}^{-1}, & x_{2} \mapsto x_{1}, \quad x_{3} \mapsto x_{3}, \quad x_{4} \mapsto x_{4}, \\
Q\left(\sigma_{2}^{-1} \sigma_{1}\right) & : & x_{1} \mapsto x_{2} * x_{1}^{-1}, \quad x_{2} \mapsto x_{3}, \quad x_{3} \mapsto x_{1} * x_{3}, \quad x_{4} \mapsto x_{4}, \\
Q\left(\sigma_{1} \sigma_{3}\right) & : & x_{1} \mapsto x_{2} * x_{1}^{-1}, \quad x_{2} \mapsto x_{1}, \quad x_{3} \mapsto x_{4} * x_{3}^{-1}, \quad x_{4} \mapsto x_{3}, \\
Q\left(\sigma_{3}\right) & : & x_{1} \mapsto x_{1}, \quad x_{2} \mapsto x_{2}, \quad x_{3} \mapsto x_{4} * x_{3}^{-1}, \quad x_{4} \mapsto x_{3} .
\end{array}
$$




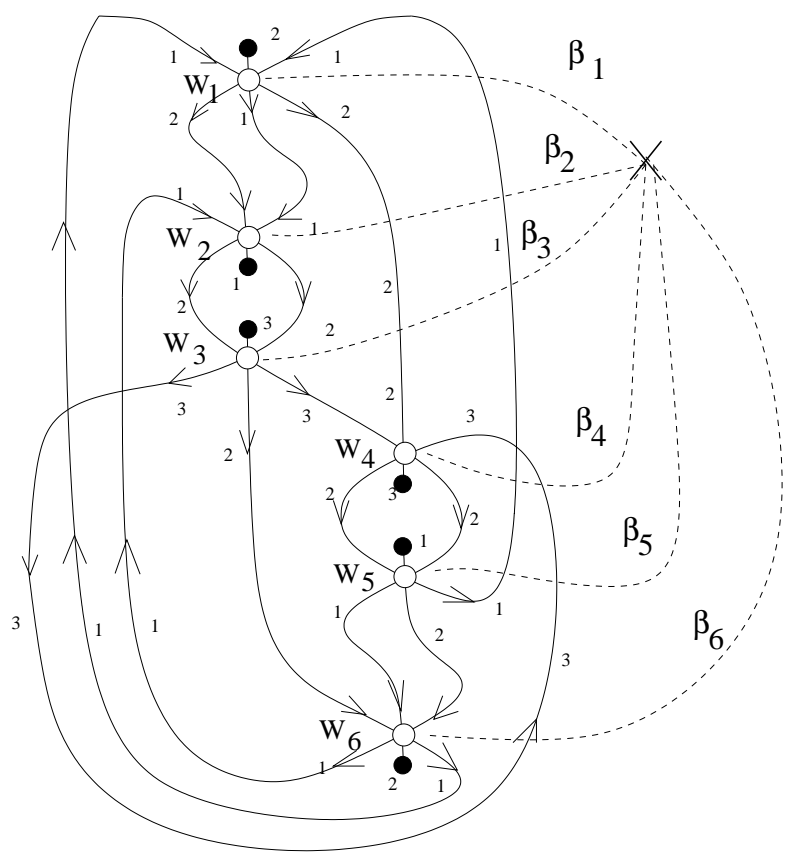

Figure 18. Computing cocycle invariants using a chart

Recall that the inclusion-induced quandle homomorphism $i_{*}: Q_{y_{0}}=F_{Q}\left\langle x_{1}, \ldots, x_{4}\right\rangle$

$\rightarrow Q(S)$ is the natural projection from $F_{Q}\left\langle x_{1}, \ldots, x_{4}\right\rangle$ to

$$
\begin{aligned}
\left\langle x_{1}, \ldots, x_{4}\right| & x_{2}=x_{1} *\left(x_{2} x_{1}\right), \\
& x_{2}=x_{2} *\left(x_{1}^{2}\right), \\
& x_{3}=x_{2}, \\
& \left.x_{4}=x_{1}\right\rangle \\
=\left\langle x_{1}, x_{2}\right| & x_{2}=x_{1} *\left(x_{2} x_{1}\right), \\
& \left.x_{2}=x_{2} *\left(x_{1}^{2}\right)\right\rangle .
\end{aligned}
$$

Then the quandle triples of the white vertices $W_{1}, \ldots, W_{6}$ are

$$
\begin{aligned}
\left(x_{2} * x_{1}^{-1}, x_{1}, x_{3}\right) & =\left(x_{1} * x_{2}, x_{1}, x_{2}\right), \\
\left(x_{2} * x_{1}^{-1}, x_{3}, x_{1} * x_{3}\right) & =\left(x_{1} * x_{2}, x_{2}, x_{1} * x_{2}\right), \\
\left(x_{3}, x_{1} * x_{3}, x_{4}\right) & =\left(x_{2}, x_{1} * x_{2}, x_{1}\right), \\
\left(x_{1}, x_{4} * x_{3}^{-1}, x_{3}\right) & =\left(x_{1}, x_{1} * x_{2}, x_{2}\right), \\
\left(x_{2} * x_{1}^{-1}, x_{1}, x_{4} * x_{3}^{-1}\right) & =\left(x_{1} * x_{2}, x_{1}, x_{1} * x_{2}\right), \\
\left(x_{1}, x_{2}, x_{4} * x_{3}^{-1}\right) & =\left(x_{1}, x_{2}, x_{1} * x_{2}\right),
\end{aligned}
$$

respectively. The signs of the white vertices are

$$
\epsilon\left(W_{1}\right)=\epsilon\left(W_{2}\right)=\epsilon\left(W_{3}\right)=+1, \quad \epsilon\left(W_{4}\right)=\epsilon\left(W_{5}\right)=\epsilon\left(W_{6}\right)=-1 .
$$


TABLE 1.

\begin{tabular}{|c|c|c|c|c|c|c|c|c|}
\hline$y_{1}$ & $y_{2}$ & $\begin{array}{c}\theta\left(y_{1} * y_{2}\right) \\
\left.y_{1}, y_{2}\right)\end{array}$ & $\begin{array}{c}\theta\left(y_{1} * y_{2},\right. \\
\left.y_{2}, y_{1} * y_{2}\right)\end{array}$ & $\begin{array}{c}\theta\left(y_{2}, y_{1} * y_{2},\right. \\
\left.y_{1}\right)\end{array}$ & $\begin{array}{c}\theta\left(y_{1}, y_{1} * y_{2},\right. \\
\left.y_{2}\right)-1\end{array}$ & $\begin{array}{c}\theta\left(y_{1} * y_{2}, y_{1},\right. \\
\left.y_{1} * y_{2}\right)-1\end{array}$ & $\begin{array}{c}\theta\left(y_{1}, y_{2},\right. \\
\left.y_{1} * y_{2}\right)^{-1}\end{array}$ & Prod \\
\hline 0 & 0 & $\begin{array}{c}\theta(0,0,0) \\
=t^{0}\end{array}$ & $\begin{array}{c}\theta(0,0,0) \\
=t^{0}\end{array}$ & $\begin{array}{c}\theta(0,0,0) \\
=t^{0}\end{array}$ & $\begin{array}{c}\theta(0,0,0)^{-1} \\
=t^{0}\end{array}$ & $\begin{array}{c}\theta(0,0,0)^{-1} \\
=t^{0}\end{array}$ & $\begin{array}{c}\theta(0,0,0)^{-1} \\
=t^{0}\end{array}$ & $t^{0}$ \\
\hline 0 & 1 & $\begin{array}{l}\theta(2,0,1) \\
=t^{0}\end{array}$ & $\begin{array}{c}\theta(2,1,2) \\
=t^{1}\end{array}$ & $\begin{array}{c}\theta(1,2,0) \\
\quad=t^{0}\end{array}$ & $\begin{array}{c}\theta(0,2,1)^{-1} \\
=t^{1}\end{array}$ & $\begin{array}{c}\theta(2,0,2)^{-1} \\
=t^{-1}\end{array}$ & $\begin{array}{c}\theta(0,1,2)^{-1} \\
=t^{0}\end{array}$ & $t^{1}$ \\
\hline 0 & 2 & $\begin{array}{c}\theta(1,0,2) \\
=t^{1}\end{array}$ & $\begin{array}{c}\theta(1,2,1) \\
=t^{0}\end{array}$ & $\begin{array}{l}\theta(2,1,0) \\
=t^{0}\end{array}$ & $\begin{array}{c}\theta(0,1,2)^{-1} \\
=t^{0}\end{array}$ & $\begin{array}{l}\theta(1,0,1)^{-1} \\
=t^{-1}\end{array}$ & $\begin{array}{c}\theta(0,2,1)^{-1} \\
=t^{1}\end{array}$ & $t^{1}$ \\
\hline 1 & 0 & $\begin{array}{l}\theta(2,1,0) \\
\quad=t^{0}\end{array}$ & $\begin{array}{c}\theta(2,0,2) \\
=t^{1}\end{array}$ & $\begin{array}{c}\theta(0,2,1) \\
=t^{-1}\end{array}$ & $\begin{array}{c}\theta(1,2,0)^{-1} \\
=t^{0}\end{array}$ & $\begin{array}{c}\theta(2,1,2)^{-1} \\
=t^{-1}\end{array}$ & $\begin{array}{c}\theta(1,0,2)^{-1} \\
=t^{-1}\end{array}$ & $t^{1}$ \\
\hline 1 & 1 & $\begin{array}{c}\theta(1,1,1) \\
=t^{0}\end{array}$ & $\begin{array}{c}\theta(1,1,1) \\
=t^{0}\end{array}$ & $\begin{array}{c}\theta(1,1,1) \\
=t^{0}\end{array}$ & $\begin{array}{c}\theta(1,1,1)^{-1} \\
=t^{0}\end{array}$ & $\begin{array}{c}\theta(1,1,1)^{-1} \\
=t^{0}\end{array}$ & $\begin{array}{c}\theta(1,1,1)^{-1} \\
=t^{0}\end{array}$ & $t^{0}$ \\
\hline 1 & 2 & $\begin{array}{c}\theta(0,1,2) \\
=t^{0}\end{array}$ & $\begin{array}{c}\theta(0,2,0) \\
=t^{1}\end{array}$ & $\begin{array}{c}\theta(2,0,1) \\
=t^{0}\end{array}$ & $\begin{array}{c}\theta(1,0,2)^{-1} \\
=t^{-1}\end{array}$ & $\begin{array}{c}\theta(0,1,0)^{-1} \\
=t^{1}\end{array}$ & $\begin{array}{c}\theta(1,2,0)^{-1} \\
=t^{0}\end{array}$ & $t^{1}$ \\
\hline 2 & 0 & $\begin{array}{l}\theta(1,2,0) \\
=t^{0}\end{array}$ & $\begin{array}{c}\theta(1,0,1) \\
=t^{1}\end{array}$ & $\begin{array}{l}\theta(0,1,2) \\
=t^{0}\end{array}$ & $\begin{array}{c}\theta(2,1,0)^{-1} \\
=t^{0}\end{array}$ & $\begin{array}{c}\theta(1,2,1)^{-1} \\
=t^{0}\end{array}$ & $\begin{array}{c}\theta(2,0,1)^{-1} \\
=t^{0}\end{array}$ & $t^{1}$ \\
\hline 2 & 1 & $\begin{array}{c}\theta(0,2,1) \\
=t^{-1}\end{array}$ & $\begin{array}{c}\theta(0,1,0) \\
=t^{-1}\end{array}$ & $\begin{array}{c}\theta(1,0,2) \\
=t^{1}\end{array}$ & $\begin{array}{c}\theta(2,0,1)^{-1} \\
=t^{0}\end{array}$ & $\begin{array}{c}\theta(0,2,0)^{-1} \\
=t^{-1}\end{array}$ & $\begin{array}{c}\theta(2,1,0)^{-1} \\
=t^{0}\end{array}$ & $t^{1}$ \\
\hline 2 & 2 & $\begin{array}{l}\theta(2,2,2) \\
=t^{0}\end{array}$ & $\begin{array}{c}\theta(2,2,2) \\
=t^{0}\end{array}$ & $\begin{array}{c}\theta(2,2,2) \\
=t^{0}\end{array}$ & $\begin{array}{c}\theta(2,2,2)^{-1} \\
=t^{0}\end{array}$ & $\begin{array}{c}\theta(2,2,2)^{-1} \\
=t^{0}\end{array}$ & $\begin{array}{c}\theta(2,2,2)^{-1} \\
=t^{0}\end{array}$ & $t^{0}$ \\
\hline
\end{tabular}

Therefore we have

$$
\begin{aligned}
\Phi_{\theta}(\Gamma)=\sum_{c} & \theta\left(c\left(x_{1} * x_{2}\right), c\left(x_{1}\right), c\left(x_{2}\right)\right) \theta\left(c\left(x_{1} * x_{2}\right), c\left(x_{2}\right), c\left(x_{1} * x_{2}\right)\right) \\
& \times \theta\left(c\left(x_{2}\right), c\left(x_{1} * x_{2}\right), c\left(x_{1}\right)\right) \theta\left(c\left(x_{1}\right), c\left(x_{1} * x_{2}\right), c\left(x_{2}\right)\right)^{-1} \\
& \times \theta\left(c\left(x_{1} * x_{2}\right), c\left(x_{1}\right), c\left(x_{1} * x_{2}\right)\right)^{-1} \theta\left(c\left(x_{1}\right), c\left(x_{2}\right), c\left(x_{1} * x_{2}\right)\right)^{-1},
\end{aligned}
$$

where $c$ runs over all possible quandle homomorphisms from $Q(S)$ to $X$. Hence

$$
\begin{aligned}
\Phi_{\theta}(\Gamma)=\sum_{y_{1}, y_{2}} & \theta\left(y_{1} * y_{2}, y_{1}, y_{2}\right) \theta\left(y_{1} * y_{2}, y_{2}, y_{1} * y_{2}\right) \theta\left(y_{2}, y_{1} * y_{2}, y_{1}\right) \\
& \times \theta\left(y_{1}, y_{1} * y_{2}, y_{2}\right)^{-1} \theta\left(y_{1} * y_{2}, y_{1}, y_{1} * y_{2}\right)^{-1} \theta\left(y_{1}, y_{2}, y_{1} * y_{2}\right)^{-1},
\end{aligned}
$$

where $y_{1}, y_{2}$ run over all elements of $X$ satisfying $y_{2}=y_{1} *\left(y_{2} y_{1}\right)$ and $y_{2}=y_{2} *\left(y_{1}^{2}\right)$.

Since the closure of $S$ in $\mathbf{R}^{4}$ is ambient isotopic to the 2-twist spun trefoil, the result follows.

11.3. Corollary. Let $\theta \in Z^{3}\left(R_{3} ; \mathbf{Z}_{3}\right)$ be the 3-cocycle

$$
t^{-\chi_{(0,1,0)}+\chi_{(0,2,0)}-\chi_{(0,2,1)}+\chi_{(1,0,1)}+\chi_{(1,0,2)}+\chi_{(2,0,2)}+\chi_{(2,1,2)},}
$$

where $R_{3}$ is the dihedral quandle of three elements, $\mathbf{Z}_{3}$ is the cyclic group $\left\langle t \mid t^{3}=1\right\rangle$ of order three, and $\chi_{(i, j, k)}$ 's are characteristic functions as before. If $F$ is the 2-twist spun trefoil, then

$$
\Phi_{\theta}(F)=3+6 t \quad \in \mathbf{Z}\left[t, t^{-1}\right] /\left(t^{3}-1\right) .
$$

Proof. Every pair $\left\{y_{1}, y_{2}\right\}$ of elements of $R_{3}$ satisfies the condition of Theorem 11.2 We have the result by a direct calculation (Table 1 will be helpful).

11.4. Theorem. Let $F^{\prime}$ be the 2-twist spun trefoil whose orientation is reversed, and $\theta$ a quandle 3 -cocycle of a finite quandle $X$ with coefficient group $A$. The state-sum invariant $\Phi_{\theta}\left(F^{\prime}\right)$ is

$$
\begin{aligned}
\sum_{y_{1}, y_{2}} \theta & \left(y_{2}, y_{1} * y_{2}, y_{1}\right)^{-1} \theta\left(y_{2}, y_{1}, y_{2}\right)^{-1} \theta\left(y_{1}, y_{2}, y_{1} * y_{2}\right)^{-1} \\
& \times \theta\left(y_{1} * y_{2}, y_{2}, y_{1}\right) \theta\left(y_{2}, y_{1} * y_{2}, y_{2}\right) \theta\left(y_{1} * y_{2}, y_{1}, y_{2}\right),
\end{aligned}
$$

where $y_{1}, y_{2}$ run over all elements of $X$ satisfying $y_{2}=y_{1} *\left(y_{2} y_{1}\right)$ and $y_{2}=y_{2} *\left(y_{1}^{2}\right)$. 
Proof. Let $S^{\prime}$ be a surface braid of degree 4 described by a 4 -chart $\Gamma^{\prime}$ in Figure 19 It is known that the closure of $S^{\prime}$ is ambient isotopic to the 2-twist spun trefoil with the reversed orientation. (In general, if an $m$-chart $\Gamma_{2}$ is a mirror image of another $\Gamma_{1}$, then the closure of a surface braid described by $\Gamma_{2}$ is ambient isotopic to the closure of a surface braid described by $\Gamma_{1}$ whose orientation is reversed.)

For a Hurwitz arc system $\mathcal{A}=\left(\alpha_{1}, \ldots, \alpha_{6}\right)$ as in Figure 19, the braid system

$$
\left(w_{1}^{-1} \sigma_{k_{1}}^{\epsilon_{1}} w_{1}, \ldots, w_{6}^{-1} \sigma_{k_{6}}^{\epsilon_{6}} w_{6}\right)
$$

of $S^{\prime}$ is given as follows:

$$
\begin{array}{ll}
w_{1}=\sigma_{1} \sigma_{3}^{-1}, & \sigma_{k_{1}}^{\epsilon_{1}}=\sigma_{2}, \\
w_{2}=\sigma_{2} \sigma_{1}^{-1} \sigma_{3}^{-1}, & \sigma_{k_{2}}^{\epsilon_{2}}=\sigma_{1}^{-1}, \\
w_{3}=\sigma_{2} \sigma_{1}^{-1} \sigma_{3}^{-1}, & \sigma_{k_{3}}^{\epsilon_{3}}=\sigma_{3}, \\
w_{4}=\sigma_{2}^{2} \sigma_{1}^{-1}, & \sigma_{k_{4}}^{\epsilon_{4}}=\sigma_{3}^{-1}, \\
w_{5}=\sigma_{2}^{2} \sigma_{1}^{-1}, & \sigma_{k_{5}}^{\epsilon_{5}}=\sigma_{1}, \\
w_{6}=1, & \sigma_{k_{6}}^{\epsilon_{6}}=\sigma_{2}^{-1} .
\end{array}
$$

The quandle automorphisms $Q\left(\sigma_{1} \sigma_{3}^{-1}\right), Q\left(\sigma_{2} \sigma_{1}^{-1} \sigma_{3}^{-1}\right), Q\left(\sigma_{2}^{2} \sigma_{1}^{-1}\right)$, and $Q(1)$ of $F_{Q}\left\langle x_{1}, \ldots, x_{m}\right\rangle$ map the generators as follows:

$$
\begin{aligned}
Q\left(\sigma_{1} \sigma_{3}^{-1}\right): & x_{1} \mapsto x_{2} * x_{1}^{-1}, \quad x_{2} \mapsto x_{1}, \quad x_{3} \mapsto x_{4}, \quad x_{4} \mapsto x_{3} * x_{4}, \\
Q\left(\sigma_{2} \sigma_{1}^{-1} \sigma_{3}^{-1}\right): & x_{1} \mapsto x_{2}, \quad x_{2} \mapsto x_{4} *\left(x_{2}^{-1} x_{1}^{-1} x_{2}\right), \\
& x_{3} \mapsto x_{1} * x_{2}, \quad x_{4} \mapsto x_{3} * x_{4}, \\
Q\left(\sigma_{2}^{2} \sigma_{1}^{-1}\right): & x_{1} \mapsto x_{2}, \quad x_{2} \mapsto x_{1} *\left(x_{2} x_{3}^{-1} x_{2}^{-1} x_{1}^{-1} x_{2}\right), \\
& x_{3} \mapsto x_{3} *\left(x_{2}^{-1} x_{1}^{-1} x_{2}\right), \quad x_{4} \mapsto x_{4}, \\
Q(1): & x_{1} \mapsto x_{1}, \quad x_{2} \mapsto x_{2}, \quad x_{3} \mapsto x_{3}, \quad x_{4} \mapsto x_{4} .
\end{aligned}
$$

Hence the defining relations $Q\left(w_{i}\right)\left(x_{k_{i}}\right)=Q\left(w_{i}\right)\left(x_{k_{i}+1}\right)(i=1, \ldots, 6)$ of $Q\left(S^{\prime}\right)$ are

$$
\begin{aligned}
x_{1} & =x_{4}, \\
x_{2} & =x_{4} *\left(x_{2}^{-1} x_{1}^{-1} x_{2}\right), \\
x_{1} * x_{2} & =x_{3} * x_{4}, \\
x_{3} *\left(x_{2}^{-1} x_{1}^{-1} x_{2}\right) & =x_{4}, \\
x_{2} & =x_{1} *\left(x_{2} x_{3}^{-1} x_{2}^{-1} x_{1}^{-1} x_{2}\right), \\
x_{2} & =x_{3} .
\end{aligned}
$$

Thus the quandle $Q\left(S^{\prime}\right)$ is

$$
\begin{array}{ll}
\left\langle x_{1}, \ldots, x_{4}\right| & x_{2}=x_{1} *\left(x_{2} x_{1}\right), \\
& x_{2}=x_{2} *\left(x_{1}^{2}\right), \\
& x_{3}=x_{2}, \\
& \left.x_{4}=x_{1}\right\rangle \\
=\left\langle x_{1}, x_{2}\right| & x_{2}=x_{1} *\left(x_{2} x_{1}\right), \\
& \left.x_{2}=x_{2} *\left(x_{1}^{2}\right)\right\rangle .
\end{array}
$$

Let $\beta_{1}, \ldots, \beta_{6}$ be the paths from points in the distinguished regions of the white vertices as in Figure [20] and let $W_{i}$ be the white vertex near the end of $\beta_{i}$ for 


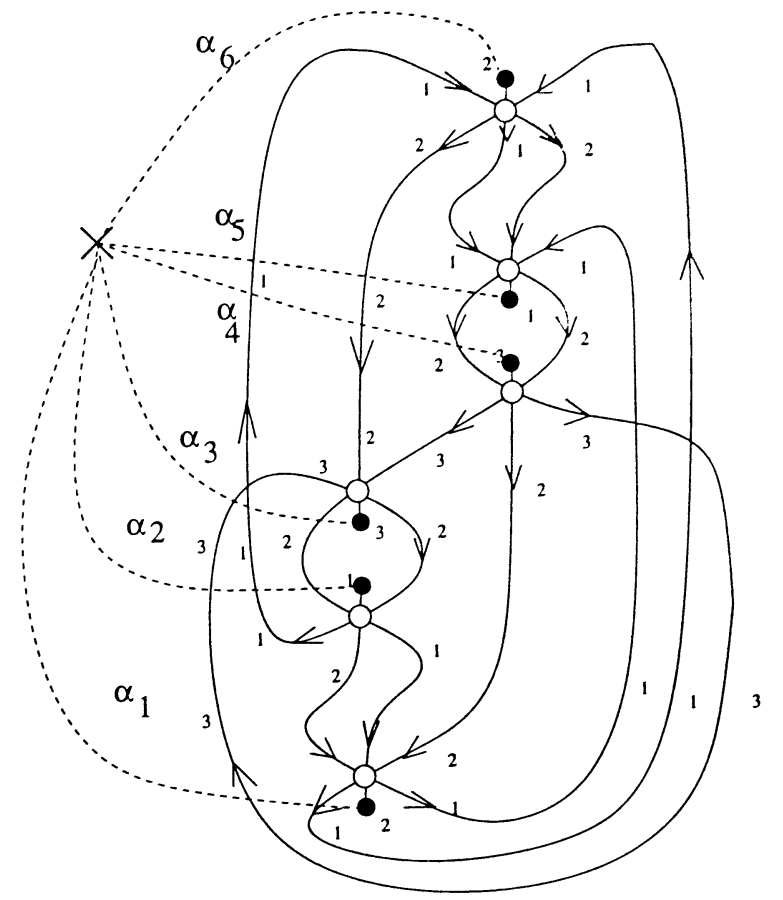

FiguRE 19. The Hurwitz system for the orientation reversed image

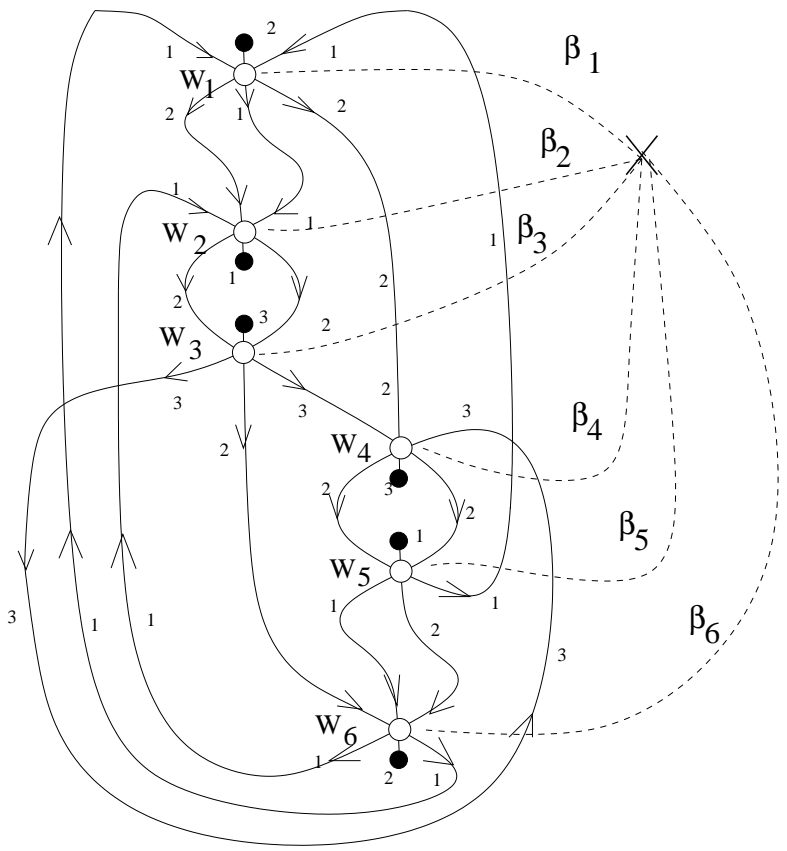

FiguRE 20. Computing cocycle invariants for the orientation reversed image 
$i=1, \ldots, 6$. The 4 -braids $\rho_{S}\left(\beta_{1}\right), \ldots, \rho_{S}\left(\beta_{6}\right)$, which are represented by the intersection braid words $w_{\Gamma}\left(\beta_{1}\right), \ldots, w_{\Gamma}\left(\beta_{6}\right)$, are

$$
\sigma_{1}^{2} \sigma_{3}^{-1}, \quad \sigma_{2}^{2} \sigma_{1}^{-1} \sigma_{3}^{-1}, \quad \sigma_{2}^{2} \sigma_{1}^{-1} \sigma_{3}^{-1}, \quad \sigma_{2}^{3} \sigma_{1}^{-1}, \quad \sigma_{2}^{3} \sigma_{1}^{-1}, \quad \text { and } \quad \sigma_{1}
$$

respectively. The quandle automorphisms $Q\left(\sigma_{1}^{2} \sigma_{3}^{-1}\right), Q\left(\sigma_{2}^{2} \sigma_{1}^{-1} \sigma_{3}^{-1}\right), Q\left(\sigma_{2}^{3} \sigma_{1}^{-1}\right)$, and $Q\left(\sigma_{1}\right)$ of $F_{Q}\left\langle x_{1}, \ldots, x_{m}\right\rangle$ map the generators as follows:

$$
\begin{aligned}
Q\left(\sigma_{1}^{2} \sigma_{3}^{-1}\right): & x_{1} \mapsto x_{1} *\left(x_{2}^{-1} x_{1}^{-1}\right), \quad x_{2} \mapsto x_{2} * x_{1}^{-1}, \\
& x_{3} \mapsto x_{4}, \quad x_{4} \mapsto x_{3} * x_{4}, \\
Q\left(\sigma_{2}^{2} \sigma_{1}^{-1} \sigma_{3}^{-1}\right): & x_{1} \mapsto x_{2}, \quad x_{2} \mapsto x_{1} *\left(x_{2} x_{4}^{-1} x_{2}^{-1} x_{1}^{-1} x_{2}\right), \\
& x_{3} \mapsto x_{4} *\left(x_{2}^{-1} x_{1}^{-1} x_{2}\right), \quad x_{4} \mapsto x_{3} * x_{4}, \\
Q\left(\sigma_{2}^{3} \sigma_{1}^{-1}\right): & x_{1} \mapsto x_{2}, \quad x_{2} \mapsto x_{3} *\left(x_{2}^{-1} x_{1}^{-1} x_{2} x_{3}^{-1} x_{2}^{-1} x_{1}^{-1} x_{2}\right), \\
& x_{3} \mapsto x_{1} *\left(x_{2} x_{3}^{-1} x_{2}^{-1} x_{1}^{-1} x_{2}\right), \quad x_{4} \mapsto x_{4}, \\
Q\left(\sigma_{1}\right): & x_{1} \mapsto x_{2} * x_{1}^{-1}, \quad x_{2} \mapsto x_{1}, \quad x_{3} \mapsto x_{3}, \quad x_{4} \mapsto x_{4} .
\end{aligned}
$$

Then the quandle triples of the white vertices $W_{1}, \ldots, W_{6}$ are

$$
\begin{aligned}
\left(x_{1} *\left(x_{2}^{-1} x_{1}^{-1}\right), x_{2} * x_{1}^{-1}, x_{4}\right) & =\left(x_{2}, x_{1} * x_{2}, x_{1}\right), \\
\left(x_{2}, x_{1} *\left(x_{2} x_{4}^{-1} x_{2}^{-1} x_{1}^{-1} x_{2}\right), x_{4} *\left(x_{2}^{-1} x_{1}^{-1} x_{2}\right)\right) & =\left(x_{2}, x_{1}, x_{2}\right), \\
\left(x_{1} *\left(x_{2} x_{4}^{-1} x_{2}^{-1} x_{1}^{-1} x_{2}\right), x_{4} *\left(x_{2}^{-1} x_{1}^{-1} x_{2}\right), x_{3} * x_{4}\right) & =\left(x_{1}, x_{2}, x_{1} * x_{2}\right), \\
\left(x_{3} *\left(x_{2}^{-1} x_{1}^{-1} x_{2} x_{3}^{-1} x_{2}^{-1} x_{1}^{-1} x_{2}\right), x_{1} *\left(x_{2} x_{3}^{-1} x_{2}^{-1} x_{1}^{-1} x_{2}\right), x_{4}\right) & =\left(x_{1} * x_{2}, x_{2}, x_{1}\right), \\
\left(x_{2}, x_{3} *\left(x_{2}^{-1} x_{1}^{-1} x_{2} x_{3}^{-1} x_{2}^{-1} x_{1}^{-1} x_{2}\right), x_{1} *\left(x_{2} x_{3}^{-1} x_{2}^{-1} x_{1}^{-1} x_{2}\right)\right) & =\left(x_{2}, x_{1} * x_{2}, x_{2}\right), \\
\left(x_{2} * x_{1}^{-1}, x_{1}, x_{3}\right) & =\left(x_{1} * x_{2}, x_{1}, x_{2}\right),
\end{aligned}
$$

respectively. The signs of the white vertices are as follows:

$$
\epsilon\left(W_{1}\right)=\epsilon\left(W_{2}\right)=\epsilon\left(W_{3}\right)=-1, \quad \epsilon\left(W_{4}\right)=\epsilon\left(W_{5}\right)=\epsilon\left(W_{6}\right)=+1 .
$$

Therefore we have

$$
\begin{aligned}
& \Phi_{\theta}\left(\Gamma^{\prime}\right)=\sum_{y_{1}, y_{2}} \theta\left(y_{2}, y_{1} * y_{2}, y_{1}\right)^{-1} \theta\left(y_{2}, y_{1}, y_{2}\right)^{-1} \theta\left(y_{1}, y_{2}, y_{1} * y_{2}\right)^{-1} \\
& \times \theta\left(y_{1} * y_{2}, y_{2}, y_{1}\right) \theta\left(y_{2}, y_{1} * y_{2}, y_{2}\right) \theta\left(y_{1} * y_{2}, y_{1}, y_{2}\right)
\end{aligned}
$$

where $y_{1}, y_{2}$ run over all elements of $X$ satisfying $y_{2}=y_{1} *\left(y_{2} y_{1}\right)$ and $y_{2}=y_{2} *\left(y_{1}^{2}\right)$. This completes the proof.

11.5. Corollary. Let $\theta \in Z^{3}\left(R_{3} ; \mathbf{Z}_{3}\right)$ be the 3-cocycle

$$
t^{-\chi_{(0,1,0)}+\chi_{(0,2,0)}-\chi_{(0,2,1)}+\chi_{(1,0,1)}+\chi_{(1,0,2)}+\chi_{(2,0,2)}+\chi_{(2,1,2)},}
$$

where $R_{3}$ is the dihedral quandle of three elements, $\mathbf{Z}_{3}$ is the cyclic group $\left\langle t \mid t^{3}=1\right\rangle$ of order three, and $\chi_{(i, j, k)}$ 's are characteristic functions as before. If $F^{\prime}$ is the 2-twist spun trefoil whose orientation is reversed, then

$$
\Phi_{\theta}\left(F^{\prime}\right)=3+6 t^{2} \quad \in \mathbf{Z}\left[t, t^{-1}\right] /\left(t^{3}-1\right) .
$$

Proof. Every pair $\left\{y_{1}, y_{2}\right\}$ of elements of $R_{3}$ satisfies the condition of Theorem 11.4 We have the result by a direct calculation (Table 2 will be helpful).

Corollaries 11.3 and 11.5 imply

11.6. Theorem. The 2-twist spun trefoil is non-invertible. 
TABLE 2 .

\begin{tabular}{|c|c|c|c|c|c|c|c|c|}
\hline$y_{1}$ & $y_{2}$ & $\begin{array}{c}\theta\left(y_{2}, y_{1} * y_{2},\right. \\
\left.y_{1}\right)-1\end{array}$ & $\begin{array}{c}\theta\left(y_{2}, y_{1},\right. \\
\left.y_{2}\right)^{-1}\end{array}$ & $\begin{array}{c}\theta\left(y_{1}, y_{2},\right. \\
\left.y_{1} * y_{2}\right)^{-1}\end{array}$ & $\begin{array}{c}\theta\left(y_{1} * y_{2}\right) \\
\left.y_{2}, y_{1}\right)\end{array}$ & $\begin{array}{c}\theta\left(y_{2}, y_{1} * y_{2},\right. \\
\left.y_{2}\right)\end{array}$ & $\begin{array}{c}\theta\left(y_{1} * y_{2},\right. \\
\left.y_{1}, y_{2}\right)\end{array}$ & Prod \\
\hline$\overline{0}$ & 0 & $\begin{array}{c}\theta(0,0,0)^{-1} \\
=t^{0}\end{array}$ & $\begin{array}{c}\theta(0,0,0)^{-1} \\
=t^{0}\end{array}$ & $\begin{array}{c}\theta(0,0,0)^{-1} \\
=t^{0}\end{array}$ & $\begin{array}{c}\theta(0,0,0) \\
=t^{0}\end{array}$ & $\begin{array}{l}\theta(0,0,0) \\
=t^{0}\end{array}$ & $\begin{array}{c}\theta(0,0,0) \\
=t^{0}\end{array}$ & $t^{0}$ \\
\hline 0 & 1 & $\begin{array}{c}\theta(1,2,0)^{-1} \\
=t^{0}\end{array}$ & $\begin{array}{c}\theta(1,0,1)^{-1} \\
=t^{-1}\end{array}$ & $\begin{array}{c}\theta(0,1,2)^{-1} \\
=t^{0}\end{array}$ & $\begin{array}{c}\theta(2,1,0) \\
=t^{0}\end{array}$ & $\begin{array}{l}\theta(1,2,1) \\
=t^{0}\end{array}$ & $\begin{array}{l}\theta(2,0,1) \\
=t^{0}\end{array}$ & $t^{2}$ \\
\hline 0 & 2 & $\begin{array}{c}\theta(2,1,0)^{-1} \\
=t^{0}\end{array}$ & $\begin{array}{c}\theta(2,0,2)^{-1} \\
=t^{-1}\end{array}$ & $\begin{array}{c}\theta(0,2,1)^{-1} \\
=t^{1}\end{array}$ & $\begin{array}{c}\theta(1,2,0) \\
=t^{0}\end{array}$ & $\begin{array}{c}\theta(2,1,2) \\
=t^{1}\end{array}$ & $\begin{array}{c}\theta(1,0,2) \\
=t^{1}\end{array}$ & $t^{2}$ \\
\hline 1 & 0 & $\begin{array}{c}\theta(0,2,1)^{-1} \\
=t^{1}\end{array}$ & $\begin{array}{c}\theta(0,1,0)^{-1} \\
=t^{1}\end{array}$ & $\begin{array}{c}\theta(1,0,2)^{-1} \\
=t^{-1}\end{array}$ & $\begin{array}{l}\theta(2,0,1) \\
=t^{0}\end{array}$ & $\begin{array}{c}\theta(0,2,0) \\
=t^{1}\end{array}$ & $\begin{array}{l}\theta(2,1,0) \\
\quad=t^{0}\end{array}$ & $t^{2}$ \\
\hline 1 & 1 & $\begin{array}{c}\theta(1,1,1)^{-1} \\
=t^{0}\end{array}$ & $\begin{array}{c}\theta(1,1,1)^{-1} \\
=t^{0}\end{array}$ & $\begin{array}{c}\theta(1,1,1)^{-1} \\
=t^{0}\end{array}$ & $\begin{array}{l}\theta(1,1,1) \\
=t^{0}\end{array}$ & $\begin{array}{l}\theta(1,1,1) \\
\quad=t^{0}\end{array}$ & $\begin{array}{l}\theta(1,1,1) \\
=t^{0}\end{array}$ & $t^{0}$ \\
\hline 1 & 2 & $\begin{array}{c}\theta(2,0,1)^{-1} \\
=t^{0}\end{array}$ & $\begin{array}{c}\theta(2,1,2)^{-1} \\
=t^{-1}\end{array}$ & $\begin{array}{c}\theta(1,2,0)^{-1} \\
=t^{0}\end{array}$ & $\begin{array}{c}\theta(0,2,1) \\
=t^{-1}\end{array}$ & $\begin{array}{c}\theta(2,0,2) \\
=t^{1}\end{array}$ & $\begin{array}{c}\theta(0,1,2) \\
=t^{0}\end{array}$ & $t^{2}$ \\
\hline 2 & 0 & $\begin{array}{c}\theta(0,1,2)^{-1} \\
=t^{0}\end{array}$ & $\begin{array}{l}\theta(0,2,0)^{-1} \\
\quad=t^{-1}\end{array}$ & $\begin{array}{c}\theta(2,0,1)^{-1} \\
=t^{0}\end{array}$ & $\begin{array}{l}\theta(1,0,2) \\
=t^{1}\end{array}$ & $\begin{array}{c}\theta(0,1,0) \\
=t^{-1}\end{array}$ & $\begin{array}{l}\theta(1,2,0) \\
=t^{0}\end{array}$ & $t^{2}$ \\
\hline 2 & 1 & $\begin{array}{c}\theta(1,0,2)^{-1} \\
=t^{-1}\end{array}$ & $\begin{array}{c}\theta(1,2,1)^{-1} \\
=t^{0}\end{array}$ & $\begin{array}{c}\theta(2,1,0)^{-1} \\
=t^{0}\end{array}$ & $\begin{array}{l}\theta(0,1,2) \\
=t^{0}\end{array}$ & $\begin{array}{l}\theta(1,0,1) \\
=t^{1}\end{array}$ & $\begin{array}{c}\theta(0,2,1) \\
=t^{-1}\end{array}$ & $t^{2}$ \\
\hline 2 & 2 & $\begin{array}{c}\theta(2,2,2) \\
=t^{0}\end{array}$ & $\begin{array}{c}\theta(2,2,2)^{-1} \\
=t^{0}\end{array}$ & $\begin{array}{c}\theta(2,2,2)^{-1} \\
=t^{0}\end{array}$ & $\begin{array}{c}\theta(2,2,2) \\
=t^{0}\end{array}$ & $\begin{array}{c}\theta(2,2,2) \\
=t^{0}\end{array}$ & $\begin{array}{c}\theta(2,2,2) \\
=t^{0}\end{array}$ & $t^{0}$ \\
\hline
\end{tabular}

Examples 10 and 11 of 15$]$ are inverses of each other. One has its Alexander ideal generated by $2 T-1$; the other has $T-2$ as the generator of the Alexander ideal. So the non-invertibility of this (Example 10/11) ribbon knot is detected by the Alexander ideal. The knot quandle, which contains the fundamental group and a choice of positive meridional element, can be used to compute the Alexander ideal. In the case of the 2-twist spun trefoil (which happens to be Example 12 of [15]), we have computed that the knotted sphere and its orientation reversed copy have the same knot quandles - thus they have the same Alexander ideal which is (non-principally) generated by $2 T-1$ and $T-2$. The invariant $\Phi_{\theta}$ is the first known state-sum invariant that detects non-invertibility of this important example.

\section{ACKNOWLEDGEMENTS}

We are grateful for a grant for visitors from Alabama EPSCoR's Mathematical Infrastructure Committee which brought Masahico Saito and Laurel Langford to Mobile for discussions. We have had valuable conversations with J. Baez, J. Birman, R. Fenn, L. Kauffman, C. Rourke, B. Sanderson, D. Ruberman, and D. Silver. José Barrionuevo, Edwin Clark, and Cornelius Pillen had helpful programming hints for the computation of quandle cocycles. Seiichi Kamada is supported by a Fellowship from the Japan Society for the Promotion of Science.

\section{REFERENCES}

[1] Baez, J. C.; Langford, L., 2-tangles, Lett. Math. Phys. 43 (1998), no. 2, 187-197. MR 2000c:57054

[2] Baez, J.; Langford, L., Higher-dimensional algebra IV: 2-Tangles, to appear in Adv. Math, preprint available at http://xxx.lanl.gov/abs/math.QA/9811139.

[3] Brieskorn, E., Automorphic sets and singularities, Contemporary math., 78 (1988), 45-115. MR 90a:32024

[4] Carter, J.S., Jelsovsky, D., Kamada, S., and Saito, M., Quandle Homology Groups, Their Betti Numbers, and Virtual Knots, Journal of Pure and Applied Algebra, 157 (2001), 135-155.

[5] Carter, J.S.; Kauffman, L.H.; Saito, M., Structures and diagrammatics of four dimensional topological lattice field theories, Advances in Math. 146 (1999), 39-100. MR 2000j:57064

[6] Carter, J.S.; Rieger, J.H.; Saito, M., A combinatorial description of knotted surfaces and their isotopies, Advances in Mathematics, 127, No. 1, April 15 (1997), 1-51. MR 98c:57023

[7] Carter, J.S.; Saito, M., Knotted surfaces and their diagrams, the American Mathematical Society, 1998. MR 98m:57027] 
[8] Carter, J.S.; Saito, M., Canceling branch points on the projections of surfaces in 4-space, Proc. AMS 116, 1, (1992) 229-237. MR 93i:57029]

[9] Dijkgraaf, R., Witten, E., Topological gauge theories and group cohomology, Comm. Math. Phys. 129 (1990), 393-429. MR 91g:81133

[10] Farber, M.S., Linking coefficients and two-dimensional knots, Soviet. Math. Doklady 16 (1975), 647-650. MR 53:4081]

[11] Farber, M.S., Duality in an infinite cyclic covering and even-dimensional knots, Math. USSRIzv. 11 (1977), 749-781. MR 58:24279

[12] Fenn, R.; Rourke, C., Racks and links in codimension two. Journal of Knot Theory and Its Ramifications Vol. 1 No. 4 (1992), 343-406. MR 94e:57006

[13] Fenn, R.; Rourke, C.; Sanderson, B., Trunks and classifying spaces, Appl. Categ. Structures 3 (1995), no. 4, 321-356. MR 96i:57023

[14] Fenn, R.; Rourke, C.; Sanderson, B., James bundles and applications, preprint available at http://www. maths. warwick.ac.uk/ cpr/ftp/james.ps.

[15] Fox, R.H., A quick trip through knot theory, in Topology of 3-Manifolds, Ed. M.K. Fort Jr., Prentice-Hall (1962) 120-167. MR 25:3522

[16] Fukuma, M., Hosono, S., and Kawai, H., Lattice topological field theory in two dimensions, Comm. Math. Phys., 161 (1994), 151-175. MR 95b:81179

[17] Giller, C., Towards a classical knot theory for surfaces in $\mathbf{R}^{4}$, Illinois Journal of Mathematics 26, No. 4, (Winter 1982), 591-631. MR 84c:57011

[18] Greene, M. T. Some Results in Geometric Topology and Geometry, Ph.D. Dissertation, Warwick (1997).

[19] Jones, V.F.R., Hecke algebra representations of braid groups and link polynomials, Ann. of Math., 126 (1989), 335-388. MR 89c:46092

[20] Joyce, D., A classifying invariant of knots, the knot quandle, J. Pure Appl. Alg., 23, 37-65. MR 83m:57007

[21] Hartley, R., Identifying non-invertible knots, Topology, 22 (1983), 137-145. MR 85c:57003

[22] Hillman, J.A., Finite knot modules and the factorization of certain simple knots, Math. Ann. 257 (1981), no. 2, 261-274. MR 83c:57009

[23] Kamada, S., Surfaces in $\mathbf{R}^{4}$ of braid index three are ribbon, Journal of Knot Theory and its Ramifications 1 (1992), 137-160. MR 93h:57039

[24] Kamada, S., A characterization of groups of closed orientable surfaces in 4-space, Topology 33 (1994), 113-122. MR 95a:57002

[25] Kamada, S., 2-dimensional braids and chart descriptions, "Topics in Knot Theory (Erzurum, 1992)," 277-287, NATO Adv. Sci. Inst. Ser. C Math. Phys. Sci., 399, Kluwer Acad. Publ., (Dordrecht, 1993).

[26] Kapranov, M.; Voevodsky, V., 2-Categories and Zamolodchikov tetrahedra equations. Proc. Symp. Pure Math., 56 (1994), Part 2, 177-259. MR 95f:18011

[27] L. H. Kauffman, Knots and Physics, World Scientific, Series on knots and everything, vol. 1, 1991. MR 93b:57010

[28] Kawauchi, A., The invertibility problem on amphicheiral excellent knots, Proc. Japan Acad., Ser.A, Math. Sci. (1979), 55, 399-402. MR 81b:57003

[29] Kawauchi, A., Three dualities on the integral homology of infinite cyclic coverings of manifolds, Osaka J. Math. 23 (1986), 633-651. MR 88e:57021

[30] Kawauchi, A., The first Alexander modules of surfaces in 4-sphere, "Algebra and Topology (Taejon, 1990)," 81-89, Proc. KAIST Math. Workshop, 5, KAIST, Taejon, Korea, 1990. MR 92b:57032

[31] Kawauchi, A., A survey of knot theory, Birkhauser, 1996. MR 97k:57011

[32] Koschorke, U., A generalization of Milnor's $\mu$-invariants to higher-dimensional link maps, Topology 36, 2 (1997), 301-324. MR 2000a:57063

[33] Langford, L., 2-tangles as a free braided monoidal 2-category with duals. Ph.D. dissertation, U.C. Riverside, 1997.

[34] Levine, J., Knot modules I, Trans. Amer. Math. Soc. 229 (1977), 1-50. MR 57:1503

[35] Matveev, S., Distributive groupoids in knot theory, (Russian) Mat. Sb. (N.S.) 119(161) (1982), no. 1, 78-88, 160. MR 84e:57008

[36] Murasugi, K., Knot theory and its applications, Translated from the 1993 Japanese original by Bohdan Kurpita. Birkheuser Boston, Inc., Boston, MA, 1996. MR 97g:57011 
[37] Neuchl, M., Representation Theory of Hopf Categories, to appear in Adv. in Math. under the title Higher-dimensional algebra VI: Hopf categories, available at http: //www . mathematik. uni-muenchen. de/ neuchl.

[38] Rolfsen, D., Knots and Links. Publish or Perish Press, (Berkley 1976). MR 58:24236

[39] Rourke, C., and Sanderson, B.J., There are two 2-twist-spun trefoils, Preprint at arxiv:math.GT/0006062.

[40] Roseman, D., Reidemeister-type moves for surfaces in four dimensional space, in Banach Center Publications 42 Knot theory, (1998) 347-380. MR 99f:57029

[41] Rosicki, Witold, Some Simple Invariants of the Position of a Surface in $\mathbf{R}^{4}$, Bull.of the Pol. Ac.of Sci. Math. 46(4), 1998, 335-344. MR 99h:57050

[42] Ruberman, D., Doubly slice knots and the Casson-Gordon invariants, Trans. Amer. Math. Soc. 279 (1983), no. 2, 569-588. MR 85e:57025

[43] Rudolph, L., Braided surfaces and Seifert ribbons for closed braids. Comment. Math. Helv. 58 (1983), no. 1, 1-37. MR 84j:57006

[44] Sanderson, B. J., Bordism of links in codimension 2, J. London Math. Soc. (2) 35 (1987), no. 2, 367-376. MR 88d:57023

[45] Sanderson, B. J., Triple links in codimension 2, Topology. Theory and applications, II (Pécs, 1989), 457-471, Colloq. Math. Soc. János Bolyai, 55, North-Holland, Amsterdam, 1993. MR 96a:57056

[46] Satoh, S., and Shima, A. The Two Twist Spun Trefiol has Triple Point Number Four, Preprint.

[47] Sekine, M., Kawauchi's second duality and knotted surfaces in 4-sphere, Hiroshima Math. J. 19 (1989), 641-651. MR 91c:57030

[48] Turaev, V., The Yang-Baxter equation and invariants of links, Invent. math. 92 (1988) 527553. MR 89e:57003

[49] Turaev, V., "Quantum invariants of knots and 3-manifolds," de Gruyter Studies in Mathematics, 18. Walter de Gruyter \& Co., (Berlin, 1994). MR 95k:57014

[50] Wakui, M., On Dijkgraaf-Witten invariant for 3-manifolds, Osaka J. Math. 29 (1992), 675696. MR 95e:57033

Department of Mathematics, University of South Alabama, Mobile, Alabama 36688

E-mail address: carter@jaguar1.usouthal.edu

Department of Mathematics, University of South Florida, Tampa, Florida 33620

E-mail address: jelsovsk@math.usf.edu

Current address: Department of Mathematics, Florida Southern College, Lakeland, Florida 33801

E-mail address: djelsovsky@flsouthern.edu

Department of Mathematics, Osaka City University, Osaka 558-8585, Japan

E-mail address: kamada@sci.osaka-cu.ac.jp

Current address: Department of Mathematics, Hiroshima University, Hiroshima 739-8526, Japan

E-mail address: kamada@math.sci.hiroshima-u.ac.jp

Department of Mathematics, University of Wisconsin at River Falls, River Falls, WISCONSIN 54022

E-mail address: laurel.langford@uwrf.edu

Department of Mathematics, University of South Florida, Tampa, Florida 33620

E-mail address: saito@math.usf.edu 